\author{
Economics Working Paper Series
}

2020/019

\title{
Forecasting Stock Returns with Large Dimensional Factor Models
}

\author{
A. Giovannelli, D. Massacci and S. Soccorsi
}

The Department of Economics

Lancaster University Management School

Lancaster LA1 4YX

UK

(C) Authors

All rights reserved. Short sections of text, not to exceed two paragraphs, may be quoted without explicit permission, provided that full acknowledgement is given. 


\title{
Forecasting Stock Returns with Large Dimensional Factor Models
}

\author{
A. Giovannelli D. Massacci \\ University of L'Aquila, Italy King's College London \\ S. Soccorsi \\ Department of Economics \\ Lancaster University Management School
}

September 14, 2020

\begin{abstract}
We study equity premium out-of-sample predictability by extracting the information contained in a high number of macroeconomic predictors via large dimensional factor models. We compare the well known factor model with a static representation of the common components with a more general model known as the Generalized Dynamic Factor Model. Using statistical and economic evaluation criteria, we empirically show that the Generalized Dynamic Factor Model helps predicting the equity premium. Exploiting the link between business cycle and return predictability, we find more accurate predictions by combining rolling and recursive forecasts in real-time, with promising results in the aftermath of the Great Financial Crisis.
\end{abstract}

JEL classification: C38, C53, C55, G11, G17.

Keywords: Stock Returns Forecasting, Factor Model, Large Data Sets, Forecast Evaluation.

\footnotetext{
${ }^{0}$ This paper benefits from comments from participants at the CORE@50 Conference (Louvain-la-Neuve, Belgium), the 36th International Symposium on Forecasting (Santander, Spain) and the Macroeconomics and Financial Time Series Analysis workshop (Lancaster, UK) and from conversations with Marc Hallin and Marco Lippi. Errors and omissions are the authors' responsibility. E-mail addresses: alessandro.giovannelli@univaq.it (Alessandro Giovannelli); daniele.massacci@kcl.ac.uk (Daniele Massacci, corresponding author); s.soccorsi@lancaster.ac.uk (Stefano Soccorsi).
} 


\section{Introduction}

Forecasting stock returns plays a key role in several areas of finance such as asset pricing, portfolio allocation and evaluation of investment managers performance: see Rapach and Zhou (2013) for a review of the literature. However, this is a challenging task: as discussed in Timmermann (2008), equity premium predictability is short-lived due to traders' searches for forecasting patterns. Early contributions conclude that out-of-sample predictability is either confined to specific periods (Pesaran and Timmermann, 1995) or completely absent (Bossaerts and Hillion, 1999; Goyal and Welch, 2003; Welch and Goyal, 2008). More recent evidence shows that returns are predictable by macroeconomic and financial variables (Campbell and Thompson, 2008; Rapach et al., 2010; Ferreira and Santa-Clara, 2011; Pettenuzzo et al., 2014; Pettenuzzo and Ravazzolo, 2016; Pan et al., 2020), and by technical indicators (Neely et al., 2014).

The majority of existing contributions study equity premium out-of-sample forecasting using a small set of predictors (see Rapach and Zhou, 2013): for example, the Welch and Goyal (2008) dataset is made of 14 and 15 variables at monthly and quarterly frequency, respectively. However, there is clear evidence of comovement and latent factor structure in large datasets of stock returns: these returns can be decomposed into common and idiosyncratic components, which are mutually orthogonal at all leads and lags; common components are driven by a small number of latent common factors, which determine comovements in the data. ${ }^{1}$ This paper studies equity premium out-of-sample forecasting using a high number of macroeconomic predictors to estimate the factors driving the comovements in returns.

Early work on factor models considered small-scale datasets: Geweke (1977), and Sargent and Sims (1977), employ exact factor models, which impose the restriction of no cross-sectional dependence on the idiosyncratic terms. On the other hand, large dimensional factor models, pioneered by Chamberlain and Rothschild (1983), rely upon an approximate factor structure, in which the idiosyncratic terms are allowed to exhibit some degree of cross-sectional dependence. More recent contributions thus study large scale information sets: see Connor and Korajczyk (1986,1988), Forni et al. (2000), Bai and Ng (2002), Stock and Watson $(2002 a, b)$, Forni et al. $(2005)$ and Forni et al. $(2015,2017)$.

We focus on the following three classes of large-dimensional factor models, which differ from each other in how they account for time series dependence in the common components, in the estimation

\footnotetext{
${ }^{1}$ See Connor and Korajczyk $(1986,1988)$.
} 
strategy, and in the forecasting equation; within each class, we then consider several specifications, which include different numbers of common factors.

(a) Stock and Watson (2002a) estimate common factors by principal components and compute predictions as projections onto the factor space. Based only on contemporaneous covariances, this is a static method for factor estimation and predictions are computed using a static representation, in which the factors are loaded contemporaneously.

(b) Forni et al. (2005) also compute predictions in a static way as projections onto the factor space. However, they allow for a data generating process with a dynamic representation known as the Generalized Dynamic Factor Model (henceforth GDFM), in which the common factors are loaded dynamically via one-sided filters (Forni et al., 2000).

(c) Forni et al. (2015, 2017) extend the dynamic method of Forni et al. (2005) by allowing for an infinite-dimensional factor space: this relaxes any restriction on the lead-lag relationships among the variables and common factors, and allows for a dynamic forecasting equation. In this sense, Forni et al. $(2015,2017)$ provide a fully fledged dynamic approach to the estimation of the GDFM.

Existing evidence on stock returns predictability with large factor models is limited to Stock and Watson (2002a) static method. Ludvingson and $\mathrm{Ng}$ (2007) find evidence of predictability in quarterly returns using a large number of macroeconomic and financial variables. At monthly frequency, Neely et al (2014) conclude that Welch and Goyal (2008) low-dimensional dataset provides valuable information to predict returns when it is augmented with technical indicators. Baetje and Menkoff (2016) find that predictability stemming from Welch and Goyal (2008) dataset is unstable and declining over time. Çakmaklı and van Dijk (2016) successfully exploit large macroeconomic information to predict monthly returns via factor augmented regressions; in a similar exercise, Gonçalves et al. (2017) find statistically significant predictability for some of the estimated factors. Ohno and Ando (2018) propose factor augmented regressions based on a shrinkage estimator.

None of the above mentioned contributions assesses the performance of the GDFM in predicting stock returns. Forni et al. (2018) extract factors from a large macroeconomic dataset similar to the one we consider in this work: their results show that the GDFM often yields more accurate predictions of macroeconomic variables than the commonly used factor model based on the static approach. Motivated 
by this encouraging result, we fill a gap in the stock return forecasting literature by contributing with the very first evidence of predictability based on the GDFM. Forni et al. (2018) focus on forecasting macroeconomic variables: our work crucially differs from theirs in that equity premium predictability patterns tend to be short-lived due to traders behavior and thus difficult to identify, as previously discussed.

We use the monthly FRED-MD large dimensional macroeconomic database of McCracken and $\mathrm{Ng}$ (2016) to conduct a pseudo real-time one-step-ahead equity premium forecasting exercise. We consider several forecasting methods (Giacomini and White, 2006; Timmermann, 2008) comprising aspects such as: the specification of the factor model (Stock and Watson, 2002a; Forni et al., 2005; Forni et al., 2015, 2017); recursive or rolling estimation windows (Timmermann, 2008); statistical and economic evaluation criteria (Leicht and Tanner, 1991; Pesaran and Timmermann, 1995). In order to facilitate comparison with the existing literature and assess the role of the macroeconomic information contained in our large dataset, we also consider the updated small-dimensional Welch and Goyal (2008) monthly dataset.

We obtain three main results. First, the information contained in large macroeconomic datasets leads to more accurate predictions both in statistical and economic terms: factor models estimated using the large-dimensional McCracken and $\mathrm{Ng}$ (2016) database outperform those that employ the smalldimensional Welch and Goyal (2008) dataset, as well as a range of small and medium-sized datasets obtained via a LASSO-driven variable selection. Second, predictions based on the GDFM, either by the estimator of Forni et al. (2005), or by that of Forni et al. (2015,2017), prevail over those based on the static method of Stock and Watson (2002a). Third, we propose a novel method selection criterion that selects the best performing method in pseudo real-time and exploits the well known cyclicality in stock returns predictability (Rapach et al., 2010) ${ }^{2}$ : this allows us to pick a model within a given class at each point in time and to timely switch between estimation windows depending on the phase of the business cycle ${ }^{3}$; the results are encouraging in the aftermath of the Great Financial Crisis. We check the robustness of our findings when real-time data, as opposed to revised data such as those in FRED-MD, are used to estimate the factors: Ghysels et al. (2018) find that bond return predictability with macro factors is an artefact generated by those revisions; we show that our results for stock return predictability

\footnotetext{
${ }^{2}$ Pesaran and Timmermann (2005) discuss the role of automated selection in forecasting. Timmermann (2008) stresses the importance of monitoring local predictability patterns for successful out-of-sample forecasting of stock returns.

${ }^{3}$ See Pesaran and Timmermann (1995), and Bossaerts and Hillion (1999), for early contributions on model selection as applied to stock returns forecasting. Clark and McCracken (2009) provide analytical, Monte Carlo, and empirical evidence of the benefits of combining estimation windows in the presence of structural breaks.
} 
are genuine and they are not an artefact due to the inclusion of pseudo real-time information.

Finally, we study the linkages between statistical and economic measures of forecast accuracy (Leicht and Tanner, 1991; Pesaran and Timmermann, 1995). We consider a risk-averse investor with meanvariance preferences and relative risk aversion parameter $\gamma$ (see Rapach and Zhou, 2013, and references therein). Our results favour the factor models of Forni et al. (2005), and Forni et al. (2015,2017); they also show that statistical and economic measures of forecast accuracy are positively correlated (Cenesizoglu and Timmermann, 2012), and that the strength of the correlation increases with $\gamma$.

The remainder of the paper is organized as follows. Section 2 provides an overview of the factor models we use in our work. Section 3 describes the data. For each model, Section 4 shows the making of estimated factors, that is the contribution of each variable and how this changes over time. Section 5 assesses the out-of-sample predictive ability of the factor models. Section 6 reports real-time results and the comparison with its pseudo real-time counterpart. Section 7 presents inferential results on the temporal pattern of forecast accuracy. Finally, Section 8 concludes.

\section{Latent factor models}

Due to the curse of dimensionality, high-dimensional modelling is a challenge for standard parametric frameworks. Latent factor modelling turns dimensionality from a curse into a blessing: it exploits the idea that the bulk of the dynamics in the data concentrates into a few latent factors, which can be recovered by aggregating an increasing number of variables of interest. The factor models we consider differ in the way such aggregation is done.

Let $\boldsymbol{x}_{t}=\left(x_{1 t}, \ldots, x_{n t}\right)^{\prime}$ be a panel of covariance stationary time series $x_{i t} \quad$ (with cross-section $i=$ $1, \ldots, n ;$ and time $t=1, \ldots, T), \boldsymbol{\Gamma}_{k}=E \boldsymbol{x}_{t} \boldsymbol{x}_{t-k}^{\prime}$ its covariance matrix with lag time $k$, and $\boldsymbol{\Sigma}(\theta)$ its spectral density matrix at frequency $\theta \in[-\pi, \pi]$. Define $\left\{v_{j}, z_{j}\right\}_{j=1}^{n}$ and $\left\{\lambda_{j}(\theta), p_{j}(\theta)\right\}_{j=1}^{n}$ the

eigenvalues (sorted in decreasing order) and the corresponding eigenvectors of $\boldsymbol{\Gamma}_{0}$ and $\boldsymbol{\Sigma}(\theta)$, respectively. Factor models imply the orthogonal decomposition

$$
x_{i t}=\chi_{i t}+\xi_{i t},
$$

where $\chi_{i t}$ is $x_{i t}$ 's common component in the sense that is driven by common factors, and $\xi_{i t}$ is its 
idiosyncratic component. Since the dynamics of the common components are driven by relatively few latent factors, the number of parameters in factor models does not increase with $n$. Consistent estimation is typically achieved as $n \rightarrow \infty$.

As the two components are mutually orthogonal at all leads and lags, the same decomposition holds true for both $\boldsymbol{\Gamma}_{k}$ and $\boldsymbol{\Sigma}(\theta)$, that is

$$
\begin{aligned}
\boldsymbol{\Gamma}_{k} & =\boldsymbol{\Gamma}_{k}^{\chi}+\boldsymbol{\Gamma}_{k}^{\xi}, \\
\boldsymbol{\Sigma}(\theta) & =\boldsymbol{\Sigma}^{\chi}(\theta)+\boldsymbol{\Sigma}^{\boldsymbol{\xi}}(\theta),
\end{aligned}
$$

where $\boldsymbol{\Gamma}_{k}^{\chi}$ and $\boldsymbol{\Gamma}_{k}^{\xi}$ are common and idiosyncratic covariances, and $\boldsymbol{\Sigma}^{\chi}(\theta)$ and $\boldsymbol{\Sigma}^{\xi}(\theta)$ are common and idiosyncratic spectral densities.

Approximate factor structures are inferred both in the time and in the frequency domain. In fact, as $n \rightarrow \infty$ we have:

(i) the number $r \ll n$ of static common factors corresponds to the number of diverging eigenvalues $v_{j}$ of $\boldsymbol{\Gamma}_{0}$ (Bai and $\mathrm{Ng}, 2002$ );

(ii) the number $q \ll n$ of dynamic common factors is equal to the number of spectral eigenvalues $\lambda_{j}(\theta)$ diverging almost everywhere in $[-\pi, \pi]$ (Hallin and Liska, 2007).

In the same way, as $n \rightarrow \infty$, idiosyncrasy is characterized by bounded idiosyncratic eigenvalues and spectral eigenvalues. ${ }^{4}$

In the rest of the paper, we refer to a dynamic estimation method for models with dynamic factors estimated considering the common-idiosyncratic decomposition of the spectral density matrix and therefore account for the whole covariance structure of the data: to this category belong the factor models described in subsections 2.2 and 2.3 below. On the other hand, the static estimation method refers to models employing static factors, which are estimated considering contemporaneous covariances only (i.e., $\boldsymbol{\Gamma}_{\mathbf{0}}$ ), rather than the whole covariance structure of the data: to this category belongs the factor model described in subsection 2.1 below.

\footnotetext{
${ }^{4}$ Therefore, limited amount of cross-sectional dependence between the idiosyncratic terms is allowed. This is the distinctive feature between the approximate factor models described here and the exact factor model studied by Geweke (1977), and Sargent and Sims (1977). In addition, serial correlation in the idiosyncratic terms is not dismissed.
} 
The most general factor model, namely the GDFM, involves the following dynamic representation for the common components

$$
\chi_{i t}=\frac{\boldsymbol{c}_{i}(L)}{\boldsymbol{d}_{i}(L)} \boldsymbol{f}_{t},
$$

where $\boldsymbol{c}_{i}(L)$ and $\boldsymbol{d}_{i}(L)$ are one-sided polynomials in the lag operator $L$ with square-summable coefficients, and $\boldsymbol{f}_{t}$ is a $q$-dimensional orthonormal white noise (see Forni et al. 2000 ) . The main advantage with respect to competing factor models is that, beyond stationarity and regularity conditions for the existence of spectral density matrix $\boldsymbol{\Sigma}(\theta)$, the GDFM does not require assumptions on the dynamics of the factor structure to achieve consistent estimation of the common components. On the contrary, the widespread static representation

$$
\chi_{i t}=\boldsymbol{\lambda}_{i}^{\prime} \boldsymbol{F}_{t},
$$

where $\boldsymbol{\lambda}_{i}$ are factor loadings, and the factors $\boldsymbol{F}_{t}$ are possibly serially correlated, imposes strong restrictions on the data generating process if consistency is to be achieved (see Hallin and Lippi, 2013 and Forni et al. 2015, 2017). Nevertheless, factor models with static representation should still be considered dynamic time series models because they can accommodate some forms of dynamics. For instance,

$$
\chi_{i t}=a_{i 1} \underbrace{\frac{f_{1 t}}{1-\alpha L}}_{F_{1 t}}+a_{i 2} \underbrace{f_{2 t}}_{F_{2 t}}+a_{i 3} \underbrace{f_{2 t-1}}_{F_{3 t}}
$$

allows for a static representation with three static factors $\left(F_{1 t}, F_{2 t}, F_{3 t}\right)$ and a dynamic representation with two dynamic factors $\left(f_{1 t}, f_{2 t}\right)$. On the contrary,

$$
\chi_{i t}=a_{i} \frac{f_{1 t}}{1-\alpha_{i} L}=a_{i}\left(f_{1 t}+\alpha_{i} f_{1 t-1}+\alpha_{i}^{2} f_{1 t-2}+\ldots\right)
$$

does not allow for a (finite-order) static representation. Notice that, while in equation (3) the impulse response functions of all common components $\chi_{i t}$ to $f_{1 t}$ only differ up to the scaling term $a_{i 1}$, in equation (4) heterogeneity is allowed in the shape of the impulse responses to $f_{1 t}$. To such dynamic heterogeneity does not correspond a finite-order static representation, as it can be seen in the term in brackets in equation (4). The model in subsection 2.3 below is based on the dynamic representation (1); those in subsections 2.1 and 2.2 below are based on the static representation (2). 


\subsection{Static method, static representation (SW)}

The method proposed by Stock and Watson (2002a), henceforth SW, involves static principal components and projections on the factor space. Let $\widehat{\boldsymbol{\Gamma}}_{0}$ be the sample counterpart of $\boldsymbol{\Gamma}_{0}$. Static factors are extracted from $\boldsymbol{x}_{t}$ by taking the $r$ principal components of $\widehat{\boldsymbol{\Gamma}}_{0}$ that solve the eigenvalue problem

$$
z_{j} \widehat{\boldsymbol{\Gamma}}_{0}=v_{j} z_{j}, \quad j=1, \ldots, r
$$

The estimated factors are $\boldsymbol{F}_{\boldsymbol{t}}^{\boldsymbol{S W}}=\widehat{\boldsymbol{z}} \boldsymbol{x}_{t}$, where $\widehat{\boldsymbol{z}}=\left(\widehat{z}_{1}, \ldots, \widehat{z}_{r}\right)^{\prime}$, and the $h$-step ahead forecast of $\chi_{i t}$ is

$$
\chi_{i t+h \mid t}^{S W}=\widehat{\boldsymbol{\Gamma}}_{h} \widehat{\boldsymbol{z}}^{\prime}\left(\widehat{\boldsymbol{z}} \widehat{\boldsymbol{\Gamma}}_{0} \widehat{\boldsymbol{z}}^{\prime}\right)^{-1} \boldsymbol{F}_{\boldsymbol{t}}^{S W}
$$

\subsection{Dynamic method, static representation (FHLR)}

The dynamic method proposed by Forni et al. (2005), henceforth FHLR, is a two-step procedure based on the dynamic estimation method and predictions formed from a static representation via a constrained projection onto the factor space.

\section{Step one: estimation}

The spectral density matrix of the data at frequency $\theta \in[-\pi, \pi]$ is estimated through discrete Fourier transforms of the sample covariance matrix

$$
\widehat{\boldsymbol{\Sigma}}(\theta)=\frac{1}{2 \pi} \sum_{k=-M}^{M} e^{-i k \theta} w_{k} \widehat{\boldsymbol{\Gamma}}_{k}
$$

where $w_{k}$ are the weights of a window function and $M$ is a truncation parameter. ${ }^{5}$

Letting $\widehat{p_{j}}(\theta)$ and $\widehat{\lambda_{j}}(\theta)$ be the eigenvector and eigenvalues of $\widehat{\boldsymbol{\Sigma}}(\theta)$, the spectral density matrices of the common and idiosyncratic components are computed as

$$
\begin{aligned}
& \widehat{\boldsymbol{\Sigma}}^{\chi}(\theta)=\sum_{j=1}^{q} \widehat{\lambda_{j}}(\theta){\widehat{p_{j}}}^{\prime}(\theta) \widehat{p_{j}}(\theta), \\
& \widehat{\boldsymbol{\Sigma}}^{\boldsymbol{\xi}}(\theta)=\sum_{j=q+1}^{n} \widehat{\lambda_{j}}(\theta){\widehat{p_{j}}}^{\prime}(\theta) \widehat{p_{j}}(\theta),
\end{aligned}
$$

\footnotetext{
${ }^{5}$ All empirical results in Section 5 are obtained using a standard triangular window.
} 
respectively; the covariances via inverse Fourier transforms are

$$
\begin{aligned}
\widehat{\boldsymbol{\Gamma}}_{k}^{\chi} & =\frac{2 \pi}{2 H+1} \sum_{j=-H}^{H} e^{i k \theta} \widehat{\boldsymbol{\Sigma}}^{\chi}\left(\theta_{j}\right), \\
\widehat{\boldsymbol{\Gamma}}_{k}^{\xi} & =\frac{2 \pi}{2 H+1} \sum_{j=-H}^{H} e^{i k \theta} \widehat{\boldsymbol{\Sigma}}^{\boldsymbol{\xi}}\left(\theta_{j}\right),
\end{aligned}
$$

with Fourier frequencies $\theta_{j}=\frac{2 \pi j}{2 H+1}$.

\section{Step two: forecasting equation}

The so-called generalized principal components of the couple $\left(\widehat{\boldsymbol{\Gamma}}_{0}^{\chi}, \widehat{\boldsymbol{\Gamma}}_{0}^{\xi}\right)$ solve the eigenvalue problem

$$
\begin{aligned}
& \widehat{z}_{j}^{g} \widehat{\boldsymbol{\Gamma}}_{0}^{\chi}=\widehat{v}_{j}^{g} \widehat{z}_{j}^{g} \widehat{\boldsymbol{\Gamma}}_{0}^{\xi}, \quad j=1, \ldots, r, \\
& \text { subject to }\left\{\begin{array}{l}
\widehat{z}_{j}^{g} \widehat{\boldsymbol{\Gamma}}_{0}^{\xi} \widehat{z}_{j}^{g}=1 \\
\widehat{z}_{i}^{g} \widehat{\boldsymbol{\Gamma}}_{0}^{\xi} \widehat{z}_{j}^{g}=0, \quad i \neq j .
\end{array}\right.
\end{aligned}
$$

Letting $\widehat{\boldsymbol{z}}^{g}=\left(\widehat{z}_{1}^{g}, \ldots, \widehat{z}_{r}^{g}\right)^{\prime}$ be a vector of the first $r$ generalized principal components, $\boldsymbol{F}_{\boldsymbol{t}}^{\boldsymbol{F} \boldsymbol{H} \boldsymbol{L} \boldsymbol{R}}=\widehat{\boldsymbol{z}}^{g} \boldsymbol{x}_{t}$ is the vector of estimated factors. ${ }^{6}$ Notice that this requires $r<\infty$, so just like the static one of SW, this estimator becomes inconsistent if the data generating process does not admit a finite dimensional static factor representation - like e.g. the model (4).

Finally, the $h$-step ahead forecast of $\chi_{i t}$ is given by

$$
\chi_{i t+h \mid t}^{F H L R}=\widehat{\boldsymbol{\Gamma}}_{h}^{\chi} \widehat{\boldsymbol{z}}^{g \prime}\left(\widehat{\boldsymbol{z}}^{g} \widehat{\boldsymbol{\Gamma}}_{0} \widehat{\boldsymbol{z}}^{g \prime}\right)^{-1} \boldsymbol{F}_{\boldsymbol{t}}^{\boldsymbol{F H} \boldsymbol{H} \boldsymbol{R}}
$$

which is a constrained projection onto the factor space since it imposes the dynamic restrictions of factor structure by using $\widehat{\Gamma}_{h}^{\chi}$ rather than $\widehat{\Gamma}_{h}$ as in the unconstrained projection (5) employed by SW.

\subsection{A fully fledged dynamic method (FHLZ)}

The method proposed by Forni et al. (2015, 2017), henceforth FHLZ, shares with FHLR the decomposition of the spectral density matrix in (6) and (7), and that of the covariances in (8) and (9), estimated

\footnotetext{
${ }^{6}$ Generalized principal components correspond to principal components of data weighted according to their signal to noise ratio.
} 
as in Step one described in subsection 2.2.

Letting $\chi_{t}^{(i)}$ be any $q+1$-dimensional subvector of common components, according to (1) it has a common factor moving average representation $\chi_{t}^{(i)}=\frac{\boldsymbol{c}^{(i)}(L)}{\boldsymbol{d}^{(i)}(L)} \boldsymbol{f}_{t}$, where $\frac{\boldsymbol{c}^{(i)}(L)}{\boldsymbol{d}^{(i)}(L)}$ is a $(q+1) \times q$-dimensional filter. Forni et al. $(2015,2017)$ prove that, since moving average representations with such "tall" filters — i.e. with more rows than columns — are generically fundamental ${ }^{7}$, they can be inverted into an autoregressive representation

$$
\boldsymbol{A}^{(i)}(L) \chi_{t}^{(i)}=\mathbf{R}^{(i)} \boldsymbol{f}_{t}
$$

where $\boldsymbol{A}^{(i)}(L)$ is $(q+1) \times(q+1), \mathbf{R}^{(i)}$ is $(q+1) \times q$, and the lag order of $\boldsymbol{A}^{(i)}(L)$ is finite and can be suitably determined. ${ }^{8}$ Let us stack all $q+1$-dimensional vectors of common components: we thus obtain an autoregressive representation in which the dynamic factors $\boldsymbol{f}_{t}$ are loaded only contemporaneously in $\boldsymbol{A}^{(i)}(L) \boldsymbol{\chi}_{t}^{(i)}{ }^{9}$ The dynamic factors can then be consistently estimated via principal components of filtered data

$$
\boldsymbol{Z}_{t}=\underline{\boldsymbol{A}}(L) \boldsymbol{x}_{t}=\underline{\boldsymbol{R}} \boldsymbol{f}_{t}+\underline{\boldsymbol{A}}(L) \boldsymbol{\xi}_{t},
$$

where $\mathbf{Z}_{t}$ collects the stacked vectors $\mathbf{z}^{(i)} \equiv \boldsymbol{A}^{(i)}(L) \chi_{t}^{(i)}, \underline{\boldsymbol{R}}$ is a tall $n \times q$ matrix and the $n \times n$ autoregressive filter takes the form

$$
\underline{\boldsymbol{A}}(L)=\left(\begin{array}{cccc}
\boldsymbol{A}^{(1)}(L) & 0 & \ldots & 0 \\
0 & \boldsymbol{A}^{(2)}(L) & & 0 \\
\vdots & & \ddots & \\
0 & 0 & \ldots & \boldsymbol{A}^{(g)}(L)
\end{array}\right) .
$$

Let $\left(\omega_{1}, \ldots, \omega_{q}\right)$ be the first $q$ eigenvalues of the covariance matrix of $\boldsymbol{Z}_{t}$, and $\Psi=\left(\psi_{1}, \ldots, \psi_{q}\right)^{\prime}$ be a $q \times n$ matrix collecting the associated eigenvectors: the estimated dynamic factors are $\boldsymbol{f}_{\boldsymbol{t}}^{\boldsymbol{F} \boldsymbol{H} \boldsymbol{L} \boldsymbol{Z}}=\Psi \boldsymbol{z}_{t}$. The estimated autoregressive coefficients in $\underline{\mathbf{A}}(L)$ are computed from the estimated common covariances

\footnotetext{
${ }^{7}$ More precisely, they are invertible because tall filters are generically zeroless. On the other hand, non-zeroless moving averages admit a multitude of nonfundamental representations which cannot be inverted into causal vector autoregressive representations (e.g., see Soccorsi, 2016). The genericity argument means that such property holds everywhere in the parameter space apart from a measure zero subset.

${ }^{8}$ In Section 4 results are obtained by determining the lag order of $\boldsymbol{A}^{(i)}(L)$ via a BIC information criterion.

${ }^{9}$ In order to avoid heavier notation, we are assuming without loss of generality that $g=n /(q+1)$; as discussed by Forni et al. $(2015,2017)$ no special challenge arises when $n$ is not a multiple of $q+1$.
} 
(8) and $\underline{\widehat{\boldsymbol{R}}}=\Psi$. Given these quantities, we have estimated impulse responses to the dynamic factors

$$
\mathbf{w}(z)=\underline{\widehat{A}(z)}^{-1} \underline{\widehat{R}}
$$

where its generic element $\mathbf{w}_{i}$ is consistent for $\frac{\mathbf{c}_{i}(z)}{\mathbf{d}_{i}(z)}$ in $(1)$, for any $i \in[1, n]$. Finally, $h-$ step ahead predictions are:

$$
\chi_{i t+h}^{F H L}=\mathbf{w}_{i h} f_{t}^{F H L Z}+\mathbf{w}_{i h-1} f_{t-1}^{F H L Z}+\ldots
$$

\section{Data}

Letting $T_{e}$ be the end point of the estimation period, our aim is to produce one-step-ahead out-of-sample forecasts for the sequence of stock returns at each point in time $\tau+1$ given the information available at time $\tau$, for $\tau=T_{e}, \ldots, T-1$. We use monthly data on stock returns along with a large set of predictors from which we estimate the factors: these are 122 variables included in the FRED-MD database described in McCracken and Ng (2016). Our data sample spans the period January 1960 to December 2014. We also use the 14 predictors originally proposed in Welch and Goyal (2008), and subsequently extended up to 2014 by the same authors: this allows for comparison with existing studies using low dimensional sets of predictors, which are reviewed in Rapach and Zhou (2013).

Stock returns are computed from the S\&P 500 index in excess of a short T-bill rate and include dividends. Formally, we let $\rho_{t+1}$ be the excess return at period $t+1$, for $t=1, \cdots, \tau-1$ : the goal is to produce one-step-ahead out-of-sample forecasts of $\rho_{\tau+1}$ given the information set available at time $\tau$, for $\tau=T_{e}, \ldots, T-1$.

The FRED-MD database organizes the variables into eight groups: (i) output and income; (ii) labor market; (iii) consumption and orders; (iv) orders and inventories; $(v)$ money and credit; (vi) interest rate and exchange rates; (vii) prices; (viii) stock market. The choice of the 122 variables was based on data availability over the period of interest as reported in the Appendix. Given the sample under consideration, we use the January 2015 vintage.

The 14 predictors proposed in Welch and Goyal (2008) are: $\log$ dividend-price ratio (log (DP)), $\log$ dividend-yield (log (DY)), log earnings-price ratio (log (EP)), log dividend-payout ratio (log (DE)), stock variance (SVAR), book-to-market ratio (BM), net equity expansion (NTIS), treasury bill rate (TBL), 
long-term yield (LTY), long-term return (LTR), term spread (TMS), default yield spread (DFY), default return spread (DFR), lagged inflation (INFL). ${ }^{10}$ As discussed in Pettenuzzo et al. (2014), the predictors fall into the following broad categories: (i) valuation ratios $(\log (\mathrm{DP}), \log (\mathrm{DY}), \log (\mathrm{EP}), \mathrm{BM}) ;($ ii $)$ measures of bond yields (TBL, LTY, TMS, DFY, DFR); (iii) estimates of equity risk (LTR, SVAR); corporate finance variables (log (DE), NTIS); (iv) macroeconomic variables (INFL).

Table 1 about here

Table 1 provides summary statistics for the series of excess stock returns and for the variables included in the Welch and Goyal (2008) dataset. Despite the difference in the sample period of interest, the figures are aligned to those displayed in Table 1 in Pettenuzzo et al. (2014).$^{11}$

As argued in Hansen and Timmermann (2012), a crucial issue in out-of-sample forecasting exercises is the choice of the sample-split between estimation and evaluation periods to avoid data mining. Following Timmermann (2008), we use the first 10 years of data as a training sample and we evaluate the forecasts over the period January 1970 to December 2014: a long evaluation sample allows for stronger power of forecast evaluation tests and minimizes the likelihood of spurious rejections. The end point $T_{e}$ of the estimation window thus is December 1969.

\section{A preliminary look at the factors}

We now look at the role that each variable in the FRED-MD dataset, as described in Section 3, plays in estimating the factors through the three models discussed in Section 2. We do so through the Degree of Commonality (hereafter DC): this measures the share of the variance of $x_{i t}$ explained by the common factors. Formally, DC is defined as

$$
D C_{i t}^{(m)}=\frac{\operatorname{var}\left(\chi_{i t}^{(m)}\right)}{\operatorname{var}\left(x_{i t}\right)}, \quad i \in[1, n], t \in\left[T_{e}+1, T\right]
$$

where $m \in(S W, F H L R, F H L Z)$ denotes the factor model, $\operatorname{var}\left(\chi_{i t}^{(m)}\right)$ is the variance of the common component estimated by the factor model $m$, and $\operatorname{var}\left(x_{i t}\right)$ is the variance of $x_{i t}$. We calculate this

\footnotetext{
${ }^{10}$ As in Welch and Goyal (2008), we lag inflation by an extra period to allow for the delay in CPI releases.

${ }^{11}$ Table 1 in Pettenuzzo et al. (2014) covers the sample period January 1927 to December 2010.
} 
measure through a rolling exercise, replicating the forecasting exercise carried out in Section 5. We present $D C_{i t}^{(m)}$ for each cross-sectional unit $i$ and time period $t$ through the heat-maps in Figure 1: the variables are divided into the groups described in Section 3; the groups are labelled in the ordinate and separated by thick horizontal red lines.

Figure 1 about here

DC displays patterns both across groups and over time. Variables included in "output and income" and in "interest and exchange rates" contribute the most to the estimated factors in all the three factor models. "Price" variables are also relevant, especially in the second half of the sample. "Money and credit" variables become important forÂăSW and FHLR factors in the second half of the sample, while their contribution remains modest for FHLZ factors .

Although the three models display clear similarities, some differences are worth discussing. On the one hand, the evolution of $D C_{i t}^{(S W)}$ resembles that of $D C_{i t}^{(F H L R)}$; on the other hand, $D C_{i t}^{(F H L Z)}$ generates somehow different paths, which may be due to the unique fully fledged dynamic form of the FHLZ estimator discussed in Section 2. For example, "output and income" variables contribute significantly to

the common factors according to $D C_{i t}^{(S W)}$ and $D C_{i t}^{(F H L R)}$; a similar pattern is observed in $D C_{i t}^{(F H L Z)}$ in most of the sample apart from the mid 90s. Similarly, "interest and exchange rate" variables play an important role over the whole sample period in the case of SW and FHLR factors; conversely, the same variables are important for FHLZ factors mainly in the central part of the sample, which approximately falls between 1980 and 2005 during the Great Moderation.

\section{Out-of-sample analysis}

\subsection{Forecasting methodology}

As in Timmermann (2008), we explicitly follow Giacomini and White (2006) and distinguish between forecasting model and forecasting method. The former refers to the underlying econometric specification, in our case the three factor models described in Section 2. The latter includes the model and other choices made by the forecaster, such as the estimator for the model unknown parameters (as discussed in Section 2), the length of the estimation window, and the evaluation criteria. 


\subsubsection{Econometric model}

We estimate the factors from the small-dimensional Welch and Goyal (2008) dataset and from the large collection of FRED-MD variables described in Section 3. In the former case, we consider $r=1,2,3$ static and $q=1,2$ dynamic factors. From the FRED-MD database, we estimate up to $r=15$ and $q=5$ static and dynamic factors, respectively: for ease of exposition and without loss of generality, we report results for $r=1,2,3,4,5,10,15$ only.

\subsubsection{Estimation window}

We consider recursive window and rolling window estimation schemes. Given the sample split described in Section 3, the former uses data from 1960:01 up to the time the forecast is made to produce a series of one-step ahead forecasts: the first forecast uses data from 1960:01 to 1969:12 to obtain an out-of-sample prediction for 1970:01; the second forecast uses data from 1960:01 to 1970:01 to produce a forecast for 1970:02, and so on. As in Timmermann (2008), the rolling window scheme employs a fixed-length window of the most recent ten years of data (i.e., 120 monthly observations) to estimate the models and produce the sequence of one-step ahead forecasts. The recursive window scheme is commonly used in the empirical literature on out-of-sample stock return forecasting: see Pesaran and Timmermann (1995), Campbell and Thompson (2008), Welch and Goyal (2008), Rapach et al. (2010), and Pettenuzzo et al., (2014). The rolling window scheme is common in the macroeconomic forecasting literature concerned with structural breaks in macroeconomic data: see Stock and Watson (2012) and Forni et al. (2018).

\subsubsection{Evaluation criteria}

As in Pesaran and Timmermann (1995), and Bossaerts and Hillion (1999), the first evaluation criterion we consider is the mean squared prediction error (MSPE), which assesses the absolute performance of a sequence of forecasts. We next compare the forecasts obtained from the factor models in relation to a given benchmark. Following Campbell and Thompson (2008), and Welch and Goyal (2008), we take as a benchmark the prevailing mean (PM), namely

$$
\rho_{t+1}=\alpha+\varepsilon_{t+1}, \quad t=1, \ldots, \tau-1, \quad \tau=T_{e}, \ldots, T-1,
$$


where $\varepsilon_{t+1}$ is a white noise error term with unpredictable mean. The equity premium forecast for period $\tau+1$ made at time $\tau$ is $\hat{\rho}_{\tau+1, \text { rec }}=\tau^{-1} \sum_{t=1}^{\tau} \rho_{t}$ under recursive window; it is equal to $\hat{\rho}_{\tau+1, \text { rol }}=$ $T_{e}^{-1} \sum_{t=\tau-T_{e}+1}^{\tau} \rho_{t}$ under rolling window. The recursive window scheme produces the benchmark usually employed in the equity premium forecasting literature: see Campbell and Thompson (2008), and Welch and Goyal (2008). As discussed in Timmermann (2008), the choice of the estimation window is a function of the underlying assumption made about the mean of the equity premium: when estimated recursively, the model in (12) assumes the equity premium has a constant mean and it is not predictable; the rolling window scheme implies that the mean of the equity premium slowly changes over time.

The MSPE may be used to measure the out-of-sample goodness of fit of a sequence of forecasts. To this purpose, we next consider the out-of-sample $R^{2}$ employed in Campbell and Thompson (2008), Timmermann (2008), Welch and Goyal (2008), Rapach et al. (2010), and Pettenuzzo et al. (2014). Let $\mathrm{MSPE}_{1}$ and $\mathrm{MSPE}_{0}$ be the mean squared prediction errors from any factor model and from the prevailing mean in (12), respectively: the out-of-sample $R^{2}$ is $R_{O o S}^{2}=1-\mathrm{MSPE}_{1} / \mathrm{MSPE}_{0}$. By construction, $R_{\text {OoS }}^{2} \leq 0$ if and only if $\mathrm{MSPE}_{1} \geq \mathrm{MSPE}_{0}$, meaning that the benchmark is at least as good as the alternative model at forecasting $\rho_{\tau+1}$; conversely, $R_{O o S}^{2}>0$ if and only if $\mathrm{MSPE}_{1}<\mathrm{MSPE}_{0}$.

Finally, we assess the statistical significance of the improvement of the alternative model over the benchmark by testing the null hypothesis $R_{O o S}^{2} \leq 0$ against the one-sided alternative $R_{O o S}^{2}>0$. We run Clark and West (2007) test (hereafter CW): this is robust to the different degrees of estimation error between models, which would otherwise favor the more parsimonious benchmark.

\subsubsection{The role of the business cycle}

Rapach et al. (2010), Henkel et al. (2011), and Rapach and Zhou (2013) argue that stock returns predictability exhibit discernible patterns linked to business cycle dynamics. We then assess our forecasts over the entire evaluation period, as well as during NBER-dated expansions and recessions. 


\subsection{Empirical results}

\subsubsection{Recursive window}

Table 2 displays the out-of-sample $R^{2}$ for the recursive window scheme.

Table 2 about here

When factors are extracted from Welch and Goyal (2008) small dimensional dataset (Panel A), the best performing model is FHLZ with $q=1$ dynamic factor: the model outperforms all other specifications over the entire evaluation period, as well as during recessions and expansions; the out-of-sample $R^{2}$ is always positive and significant at $5 \%$ level or less. ${ }^{12}$ The out-of-sample $R^{2}$ is equal to $0.95 \%$ over the whole evaluation period, and to $0.75 \%$ and $1.39 \%$ during expansions and recessions, respectively. The forecasts produced by FHLZ with $q=1$ dynamic factor are thus more accurate during contractionary periods: this is consistent with Campbell and Cochrane (1999), Menzly et al. (2004), and Bekaert et al. (2009), who argue that risk premia are countercyclical and drive (at least part of) predictability; it also resembles Rapach et al. (2010), Henkel et al. (2011), and Rapach and Zhou (2013), who empirically show that out-of-sample stock returns predictability increases during recessions as compared to expansions.

Panel B in Table 2 shows that when the whole large dimensional FRED-MD dataset is used to estimate the factors, the best performing model is FHLR. The specifications with $r=2,3$ static factors and $q=1$ dynamic factor overall produce the most accurate forecasts, with statistically significant improvements over the prevailing mean at $10 \%$ level or less. These two models also outperform FHLZ with $q=1$ dynamic factor estimated from the small-dimensional Welch and Goyal (2008) dataset (see Panel A): with the data at hand, large dimensional factor models provide a hedge over smaller scale counterparts.

Boivin and $\mathrm{Ng}$ (2006) question whether adding series with little factor structure to estimate factors may result in factors being less useful for out-of-sample forecasting purposes. Panels C, D and E in Table 2 show results for the same models as in Panel B, but where factors are estimated from 25, 50 and 75 series from the FRED-MD dataset, respectively: the series are selected through a pseudo real-time LASSO

\footnotetext{
${ }^{12}$ As stressed in the footnote 19 of Pettenuzzo et al. (2014), the $p$-values from the Clark and West (2007) test should be interpreted with caution and in line with Diebold (2015): those $p$-values should be intended to compare forecasts rather than models.
} 
procedure at each time $\tau$ the forecast is made. When only a subset of the series is used to estimate the factors, the forecasting ability of the models, as measured by the out-of-sample $R^{2}$, deteriorates. The performance of the forecasts improves as more series are used to estimate the factors. This result shows the usefulness of large data and is in line with basic asymptotic results on consistent factor estimation, which is achieved for growing cross-sections.

Given the data at hand, large dimensional datasets are more informative than small-dimensional counterparts in a recursive window framework; FHLR models overall produce the most accurate forecasts with $r=2,3$ static factors and $q=1$ dynamic factor.

\subsubsection{Rolling window}

Table 3 displays the out-of-sample $R^{2}$ for the rolling window scheme.

Table 3 about here

As with the recursive window, factor models estimated using the large dimensional FRED-MD dataset (see Panel B) generally produce more accurate forecasts than those based on the small scale Welch and Goyal (2008) dataset (see Panel A). Over the whole forecasting period, FHLZ forecasts are the most precise: the out-of-sample $R^{2}$ ranges between $1.17 \%$ and $2.02 \%$, and it is always statistically greater than zero at least at $5 \%$ level. During expansionary periods, no large dimensional model unambiguously dominates any other specification: in particular, FHLZ with $q=2$ dynamic factors as obtained from the Welch and Goyal (2008) dataset has the highest out-of-sample $R^{2}$ out of all models, which is equal to $0.54 \%$ and it is significant at $10 \%$ level. During economic recessions, FHLR and FHLZ models combined with the large dimensional FRED-MD dataset produce forecasts of similar accuracy: almost all out-of-sample $R^{2}$ are positive and significant at least at $10 \%$ level. The forecasts from SW models are statistically significant for low values of the number of static factors $r$, although they are always negative. When factors are estimated from a subset of 25,50 and 75 variables from the FRED-MD dataset selected using a LASSO type procedure (see Panels C, D and E, respectively), the quality of the forecasts deteriorates; as in the case of the recursive window scheme, the precision of the forecasts improves as more variables are used to estimate the factors.

In conclusion, the results from the rolling window scheme generally favor large dimensional factor 
models, with FHLR and, especially, FHLZ having a hedge over SW.

\subsubsection{The role of large macroeconomic information}

In order to asses the role of our large cross-section of macroeconomic information, in Table 4 we resume the LASSO results in Tables 2 and 3 by picking the best specifications of SW, FHLR, FHLZ for each level of variable selection (i.e., 25,50 , and 75 variables) under both estimation windows.

Table 4 about here

Comparing the results from the cross-sections restricted by the LASSO with those from the full FREDMD dataset, we observe an almost monotonic improvement in the number of included variables, as evidenced by the increasing out-of-sample $R^{2}$. In line with standard asymptotic results on factor models as discussed in Section 2, this empirical finding suggests that the underlying assumptions are likely to hold in the data at hand and the models we consider are not misspecified. This confirms the conclusion of McCracken and Ng (2016), who propose the FRED-MD dataset as a resource for factor analysis. Stock and Watson (2012), and Giannone et al. (2017), find similar results regarding the performance of shrinkage methods as applied to forecasting problems where large-dimensional macroeconomic datasets are used.

\subsection{An adaptive method selection approach}

The results discussed in Sections 5.2.1 and 5.2.2 and displayed in Tables 2 and 3, respectively, show two important findings: factor models estimated using large dimensional datasets tend to produce more reliable forecasts than those estimated using a smaller number of macroeconomic series; models based on the dynamic method (i.e., FHLR and FHLZ) outperform SW, which is based on the static method. These findings come from a high number of forecasting methods, as discussed in Section 5.1.

We also find an empirical regularity along the business cycle: rolling forecast are more accurate during recessions, while recursive forecasts have an edge during expansions. As argued in Pesaran and Timmermann (2005), decision makers require selecting the best performing method in real-time. We thus implement what we label a method selection criterion: in the spirit of Pesaran and Timmermann (1995), and Bossaerts and Hillion (1999), this allows us to pick a model within a given class at each point 
in time; it further allows us to timely switch between estimation windows, whose importance is stressed in Clark and McCracken (2009), and Pesaran and Timmerman (2007). We can thus exploit more fully the cyclical behaviour in returns predictability discussed in Rapach and Zhou (2013).

\subsubsection{Model selection strategy}

In the spirit of Pesaran and Timmermann (1995), and Bossaerts and Hillion (1999), we study the model selection problem within SW, FHLR and FHLZ for a given estimation window.

When implementing model selection criteria using the recursive window scheme, we have to account for structural instability in the underlying factor model: see Baltagi et al. (2017), and references therein. We adopt the following strategy to tackle the problem of model selection in the presence of structural instability under the recursive window scheme. As suggested in Stock and Watson (2012), we a priori select $r=5$ static factors and we keep this number fixed over the entire out-of-sample evaluation period. To the very best of our knowledge, no existing study allows us to a priori fix the number of dynamic factors. At each point in time, we choose $q=4$ dynamic factors using Hallin and Liška (2007) criterion as applied to the rolling window scheme: model instability is less likely to affect this estimation scheme as the dynamic window effectively adapts to time variation in the loadings. ${ }^{13}$

The empirical results with $r=5$ and $q=4$ show that FHLR and FHLZ outperform SW (see Panel B in Table 2): the former two produce forecasts with higher out-of-sample $R^{2}$ than the latter during the entire out-of-sample evaluation period, as well as during expansions and recessions. Between the two dynamic models, FHLZ generates more accurate forecasts than FHLR over the full evaluation period and in expansionary phases: the out-of-sample $R^{2}$ is equal to $0.75 \%$ and $0.56 \%$, respectively, and in both cases it is significant at $5 \%$ level. During recessions, the out-of-sample $R^{2}$ from FHLZ forecasts is marginally higher than that from FHLR forecasts; in the latter case, statistical significance is achieved at $10 \%$ level. Overall, FHLZ has an edge over FHLR under the recursive window scheme.

Under rolling window estimation, at each point in time we choose the number of static and dynamic factors according to Bai and Ng (2002), and Hallin and Liška (2007) criteria, respectively: the problem of structural instability is likely to be less relevant in this case, as rolling window estimation accounts for time-variation in the parameters. As with the recursive window scheme, FHLR and FHLZ fare better

\footnotetext{
${ }^{13}$ At each point in time, Hallin and Liška (2007) criterion selects between $q=4$ and $q=5$ dynamic factors. We choose $q=4$ as a matter of parsimony. Results with $q=5$ are very similar and are available upon request.
} 
than SW (see Panel B in Table 3). Between the two dynamic models, FHLZ forecasts are better during the whole evaluation period: the out-of-sample $R^{2}$ is equal to $1.71 \%$ and significant at $5 \%$ level. Rolling forecasts from FHLR are more accurate during recessions: the higher out-of-sample $R^{2}$ is $5.36 \%$ and significant at $5 \%$ level.

\subsubsection{Switching the estimation window in pseudo real-time}

As shown in Paye and Timmermann (2006), and Rapach and Wohar (2006), return prediction models are subject to structural instabilities. Clark and McCracken (2009), and Pesaran and Timmerman (2007), argue that forecast accuracy in the presence of breaks may be improved by combining recursive and rolling estimation windows in such a way that optimally handles the trade-off between variance (which decreases with the sample size and so in recursive windows) and bias (which is generated by the breaks and so is less harmful within rolling windows). Inoue et al. (2017) develop a procedure to determine the window size in the presence of structural instability. Based on our empirical findings, we propose to select the estimation window as a function of business cycle conditions so that forecast accuracy can enhance in the presence of instabilities linked to the business cycle. This is relevant as out-of-sample stock returns predictability depends on the business cycle, as stressed in Rapach et al. (2010).

Table 5 about here

In order to empirically motivate our strategy, Table 5 reports mean squared prediction errors (multiplied by 100) for the three large dimensional factor models we consider under recursive and rolling window estimation (Panels A and B, respectively). We first look at the whole set of available factor models. During economic expansions, all models produce better forecasts under recursive window estimation. The scenario changes during contractionary periods: forecasts from SW models have similar MSPE under recursive and rolling windows; FHLR and FHLZ models produce more accurate forecasts under rolling window. This finding is confirmed when the model selection strategy detailed in Section 5.3.1 is applied within each class of factor models: this a priori selects $r=5$ and $q=4$ under recursive window; it resorts to Bai and Ng (2002), and Hallin and Liška (2007) criteria under rolling window. The results confirm that FHLR and FHLZ generate better forecasts under recursive and rolling window during expansions and recessions, respectively. This last finding suggests that timely switching between 
estimation windows may improve the quality of the forecasts.

In order to select the estimation window, we follow Banbura et al. (2011) and employ a nowcasting procedure that tracks the current state of the economy. At each point in time $\tau$, we use the sequence $\left\{\operatorname{ADS}_{t}\right\}_{t=1}^{\tau}$ of business cycle indicators of Aruoba et al. (2009) and select the estimation window by solving

$$
\hat{\theta}_{\tau}=\arg \min _{\theta}\left|\left[\tau^{-1} \sum_{t=1}^{\tau} \mathbb{I}\left(\operatorname{ADS}_{t}<\theta\right)\right]-R\right|, \quad \tau=T_{e}, \ldots, T-1,
$$

where $\mathbb{I}(\cdot)$ denotes the indicator function and $|\cdot|$ the absolute value of the argument: $R=0.14$ is the approximate sample frequency of recessions over the $1946: 01-1969: 12$ period as identified by the NBER business cycle dates. ${ }^{14}$ At each point in time $\tau$, the threshold $\hat{\theta}_{\tau}$ minimizes the distance between the empirical frequency $R$ and the one identified by Aruoba et al. (2009) business cycle indicator; for each $\tau$, we select recursive and rolling window if $\operatorname{ADS}_{\tau} \geq \hat{\theta}_{\tau}$ and $\operatorname{ADS}_{\tau}<\hat{\theta}_{\tau}$, respectively.

Table 6 about here

Results related to the proposed model selection criterion are displayed in Table 6 . The table collects results for the PM model in (12), and for the large dimensional factor models SW, FHLR and FHLZ: under recursive and rolling windows, factor models are estimated as detailed in Sections 5.2.1 and 5.2.2, respectively; the model selection criterion chooses the estimation window according to the procedure previously described in this section. The results show that the mean squared prediction error (multiplied by 100) for FHLR and FHLZ is minimized when the method selection criterion is implemented and it is equal to 0.1981 and 0.1967 , respectively (see Panel A): these value are lower than any other obtained from PM and SW. Table 6 also calculates the out-of-sample $R^{2}$ with respect to the most accurate benchmark, namely PM estimated by recursive window (Panel B): SW delivers negative values regardless of the estimation window; FHLR forecasts obtained from the method selection criterion outperform the benchmark with a positive out-of-sample $R^{2}$ equal to $1.11 \%$; FHLZ produces the most accurate forecasts, which always deliver positive values for the out-of-sample $R^{2}$, with highest value equal to $1.80 \%$ achieved under the proposed method selection procedure.

\footnotetext{
${ }^{14}$ We also considered the case in which the empirical frequency of recessions at time $\tau$ is determined by the expanding window between 1946: 01 and $\tau$. The results are quantitatively very similar to those from the case $R=0.14$ and are available upon request.
} 


\subsubsection{Statistical forecast accuracy and portfolio choice}

Following the pioneering work of Leicht and Tanner (1991), and Pesaran and Timmermann (1995), we finally study the economic value of equity premium forecasts. Our interest lies in understanding the linkages between statistical and economic measures of forecasting performance. This is an open issue: Leicht and Tanner (1991), and Cenesizoglu and Timmermann (2012), only find weak relationships between statistical and economic measures of forecast accuracy; at the same time, Pesaran and Granger (2000) advocate a closer link between decision theory and the forecast evaluation problem.

In line with Campbell and Thompson (2008), Rapach et al. (2010), Ferreira and Santa Clara (2011), Rapach and Zhou (2013) and Neely et al. (2014), we economically evaluate the forecasts by computing the certainty equivalent return for a risk-averse investor with mean-variance preferences and relative risk aversion parameter $\gamma$. At the end of each month, the investor allocates her wealth between stocks and a riskless asset. The choice depends on a dynamic trading strategy based on a benchmark and an alternative prediction method. As customary in the literature, our benchmark is the prevailing mean estimated with recursive window: as pointed out in Timmermann (2008), it assumes the equity premium has a constant mean and it is not predictable; it also produces the lowest mean squared prediction error out of all methods based on the prevailing mean (see Panel A in Table 6).

Formally, let $j=0$ and $j=1$ denote the benchmark and the alternative method, respectively. If the investor opts for method $j$ at period $\tau+1$, at period $\tau$ she assigns to stocks a share $w_{j \tau}$ equal to

$$
w_{j \tau}=\frac{1}{\gamma} \frac{\hat{\rho}_{j, \tau+1}}{\hat{\sigma}_{j, \tau+1}^{2}}, \quad j=0,1
$$

where $\hat{\rho}_{j, \tau+1}$ and $\hat{\sigma}_{j, \tau+1}^{2}$ are the point forecasts of $\rho_{\tau+1}$ and of its variance $\sigma_{\tau+1}^{2}$ made at time $\tau$, respectively: as in Campbell and Thompson (2008), we compute the latter as the five-year moving window of past monthly returns, so that $\hat{\sigma}_{j, \tau+1}^{2}=\hat{\sigma}_{\tau+1}^{2}$ is independent of the underlying forecasting method $j$. The realized return on the investment portfolio from method $j$ at time $\tau+1$ then is

$$
R_{j, \tau+1}^{p}=w_{j \tau} \rho_{\tau+1}+r_{f \tau}, \quad \tau=T_{e}, \ldots, T-1, \quad j=0,1
$$

The certainty equivalent return is the average realized utility from method $j$ over the out-of-sample 
period and it is defined as

$$
\bar{U}_{j}=\bar{\mu}_{j}^{p}-\frac{1}{2} \gamma\left(\hat{\sigma}_{j}^{p}\right)^{2}, \quad j=0,1
$$

where $\bar{\mu}_{j}^{p}$ and $\left(\hat{\sigma}_{j}^{p}\right)^{2}$ are the sample mean and variance, respectively, of the portfolio returns $R_{\tau, t+1}^{p}$ over the out-of-sample period. Following Campbell and Thompson (2008), we constrain the portfolio weights $w_{0 \tau}$ and $w_{1 \tau}$ such that $0 \leq w_{0 \tau}, w_{1 \tau} \leq 1.5$. We then compute the utility gain

$$
\Delta=\bar{U}_{1}-\bar{U}_{0}:
$$

as discussed in Fleming et al. (2001), the utility gain represents the portfolio management performance fee that a mean-variance investor is willing to pay to switch from the dynamic trading strategy based on the benchmark to the one based on the alternative method. In the empirical application, we set $\gamma=3,4,5,10$ : these are aligned to the values chosen in Rapach et al. (2010), Rapach and Zhou (2013), and Cenesizoglu and Timmermann (2012). We then multiply $\Delta$ by 1200 to express it in average annualized percentage returns.

The results are displayed in Panels C, D, E and F of Table 6 for $\gamma=3,4,5,10$, respectively. The recursive window estimation scheme leads to less accurate forecasts than those produced by the benchmark uniformly across models as all utility gains are negative. Interesting results arise under the rolling window scheme and the method selection criterion. The most accurate forecasts are produced by FHLR and FHLZ: the former prevails for $\gamma=3,4$ and $\gamma=5$ under rolling window and method selection, respectively; the latter is preferable for $\gamma=10$ under method selection. The empirical results indicate that the link between statistical and economic measures of forecast accuracy increases with risk aversion. An analysis of the correlation between out-of-sample $R^{2}$ and utility gains confirms this first impression: the correlation is equal to $-0.03,0.10,0.19$ and 0.46 for $\gamma=3,4,5,10$, respectively, and thus monotonically increases in $\gamma$. This empirical regularity is further illustrated in Figure 2 , which plots utility gains against out-of-sample $R^{2}$ for the values of $\gamma$ of interest.

\section{Figure 2 about here}

In conclusion, our results show that for the empirically relevant values $\gamma=3,4,5$, FHLR produces the most accurate forecasts as evaluated in economic terms: rolling window estimation is preferable for $\gamma=3,4$, whereas the proposed method selection criterion takes the lead for $\gamma=5$. In the extreme case 
$\gamma=10$, the method selection criterion as applied to FHLZ should be employed. As in Cenesizoglu and Timmermann (2012), we further show that statistical and economic measures of forecast accuracy tend to be positively correlated; in addition, the strength of the correlation increases with $\gamma$.

\section{Real-time evidence}

The results in Section 5 are based on the FRED-MD dataset: since the macroeconomic variables in FRED-MD are observed at their final vintage after multiple revisions, those results come from a pseudo real-time forecasting exercise. As noticed in Ghysels et al. (2018), data revisions affect bond return predictability: we investigate whether we face a similar issue in forecasting stock returns with large dimensional factor models.

We assess the robustness of our main findings by running an analysis similar to the one in Section 5 using data that are available in real-time. We employ a dataset of 62 variables collected from the St. Louis Fed ALFRED data archive: the sample starts in February 1982 and ends in December 2014. ${ }^{15}$ The ADS business cycle indicator we use to combine rolling and recursive forecasts according to the method selection criterion described in Section 5.3 is also subject to data revisions: for this reason, we consider its real-time version. ADS vintage data are provided by the Federal Reserve of Philadelphia and are available starting from $2009 .{ }^{16}$ Due to real-time data availability, rolling and recursive forecasts are evaluated between February 1992 to December 2014, whereas forecast combinations obtained using the method selection criterion begin in January 2009.

The sample for real-time analysis is a subsample of that available for the pseudo real-time exercise conducted in Section 5: for a meaningful comparison, we also report the results from the pseudo real-time exercise over the sample period that begins in 2009. Table 7 compares the results in terms of MSPE and out-of-sample $R^{2}$ : panels $\mathrm{A}$ and $\mathrm{B}$ refer to the exercise with data in pseudo real-time; panels $\mathrm{C}$ and $\mathrm{D}$ show the results obtained using only information available in real-time. Recursive window forecasts are less accurate than those produced by the benchmark uniformly over all models when real-time data are involved: these results are in line with the findings from pseudo real-time data with the exception of those

\footnotetext{
${ }^{15}$ ALFRED data are available at https://alfred.stlouisfed.org. In the construction of the dataset here employed, we discarded time series therein for which too few vintages are available together with other irregular time series. More details on the ALFRED dataset are provided in the Appendix.

${ }^{16}$ All ADS data are available at https://www.philadelphiafed.org/research-and-data/real-time-center/ business-conditions-index.
} 
obtained from FHLZ, which produces enhanced forecasts with respect to the benchmark when revised data are employed. Under rolling window, SW and FHLR underperform with respect to the benchmark with real-time data, whereas FHLZ becomes more accurate. Finally, when the method selection criterion is used, real-time and pseudo real-time information produce qualitatively similar results.

Table 7 about here

The results in Table 7 let us conclude that there is no sizeable difference in predictive accuracy when real-time, as opposed to revised, data are used for factor estimation and return forecasting. It should also be noticed that ALFRED and FRED-MD are datasets of different cross-sectional dimensions, being made of 62 and 131 variables, respectively. Other conditions being equal, this difference in the cross-sectional dimension between the two datasets would a priori predict a relative deterioration in factor estimation when ALFRED is involved. In fact, such a deterioration is not observed in our empirical findings: unlike those in Ghysels et al. (2018) on bond returns, our results show that stock return predictability does not deteriorate when real-time data are employed; a possible explanation is that bond and equity markets react differently to macroeconomic news, as argued in Andersen et al. (2007).

\section{$7 \quad$ Local predictability}

Following Farmer et al. (2019), we now study local predictability, as opposed to global predictability, which was the focus of the analysis up to now. In order to perform time-varying inference, we employ the test proposed by Giacomini and Rossi (2010) (hereafter GR): this allows us to investigate how the predictability provided by our forecasting methods distributes over time with respect to the benchmark. GR test results on the forecasts produced by the selected specifications of SW, FHLR and FHLZ are reported in Figures 3, 4, 5, respectively. The solid lines correspond to the GR test statistics, which is the normalized and smoothed difference between the square forecast errors of a given factor model, estimated with one of the methods considered, and the benchmark; the smoothing considered is the centred moving average of 60 observations corresponding to 5 years of forecast. The zero horizontal line indicates equal performance, the dotted lines indicate the $5 \%$ critical values, so that the factor model considered outperforms (underperforms) the benchmark locally in time at the $5 \%$ significance level when 
the solid line is below (above) the lower (upper) dashed line.

Forecasts based on FRED-MD data are displayed in the left-hand side columns of Figures 3, 4, 5; recursive and rolling predictions are the top and central plots, respectively. All models significantly outperform the benchmark during the first decade of our sample, regardless of whether the estimation window is recursive or rolling. The last decade of our sample is also associated with some predictability: this is obtained with the rolling window and is caught for a sustained period by FHLZ, while it is very short-lived in the case of SW and FHLR forecasts. SW rolling and FHLR (either rolling and recursive) outperform the benchmark at some point in the late 80 s, although only for a short period of time.

Figures 3, 4, 5 about here

We present GR test results for SW, FHLR and FHLZ forecast combinations based on our method selection criterion in the bottom-left plots of Figures 3, 4, 5, respectively. The figures show that the method selection criterion yields some promising results in the aftermath of the Great Financial Crisis. By comparing our forecast combinations in the bottom-left plots with recursive and rolling forecasts in the top-left and centre-left plots, respectively, we can see that the improvements within that time period are evident for all the factor models: FHLR predictions are significantly more accurate than the benchmark for a relatively long time after the crisis; our method selection criterion as applied to FHLZ produces significantly more accurate forecasts for a prolonged period despite the fact that the underlying recursive and rolling forecasts only infrequently outperform the benchmark; SW recursive and, less frequently, SW rolling are significantly outperformed by the benchmark, whereas SW combined predictions are as accurate as the benchmark.

Finally, we compare the pseudo real-time forecasts based on the revised FRED-MD data with the real-time counterparts obtained from ALFRED, the latter being displayed in the right-hand side columns of Figures 3,4 and $5 .{ }^{17}$ The results show no noticeable difference when the forecasts are generated using either SW or FHLR. In line with the results discussed in Section 6, in the case of the FHLZ model estimated with rolling window, real-time data generate more accurate forecasts than when pseudo realtime data are employed.

\footnotetext{
${ }^{17}$ For the plots at the bottom-right, corresponding to the exercise based on vintages of both the ADS business cycle indicator and the data used to extract the factors (i.e. ALFRED), due to the limited number of forecasts we implemented the test with a centered average of 24 observations. Indeed, consistency in the kernel estimation underlying the GR test requires the usual asymptotic properties of the smoothing window which is supposed to grow (less than linearly) with the sample size.
} 


\section{Conclusions}

We study one-step-ahead out-of-sample predictability of the monthly equity premium using large dimensional factor models. We compare the static method of Stock and Watson $(2002 a, b)$ with the more general approach known as Generalized Dynamic Factor Model, for which the two estimators proposed in Forni et al. (2005), and Forni et al. (2015, 2017), are considered. Through statistical and economic forecast evaluation criteria, we show that large dimensional factor models condense the information contained in a high number of predictors to accurately forecast stock returns, especially when the Generalized Dynamic Factor Model is considered. Further improvements are found by applying a combination of recursive and rolling forecasts, which we label method selection: this combines information stemming from both windows, depending on the underlying state of the economy; it provides promising results in the aftermath of the Great Financial Crisis. Our conclusions are unaffected by data revisions as a real-time predictive exercise yields similar results to its pseudo real-time counterpart.

Our work may be extended in several ways: three of them are worth discussing. Barigozzi et al. (2019) study a two-step Generalized Dynamic Factor Model for volatilities, which also accounts for the factor structure in returns: it would be worth exploring whether our method selection criterion delivers more accurate volatility predictions within that framework. More generally, it would be interesting to study how well large dimensional factor models predict the conditional distribution of equity returns following an approach similar to that in Massacci (2015). Finally, we focused on stock returns: the analysis of bond returns predictability is high in our research agenda (see Gargano et al., 2017, and references therein).

\section{References}

[1] Andersen, T.G., Bollerslev, T., Diebold, F.X., C.Vega (2007), "Real-time price discovery in global stock, bond and foreign exchange markets", Journal of International Economics, 73, 251-277.

[2] Aruoba, S. B., F. X. Diebold, and C. Scotti (2009), "Real-Time Measurement of Business Conditions," Journal of Business \& Economic Statistics, 27 (4), 417 - 427.

[3] Baetje, F., and L. Menkhoff (2016), "Equity Premium Prediction: Are Economic and Technical Indicators Unstable?", International Journal of Forecasting, 32(4), 1193 - 1207. 
[4] Bai, J., and S. Ng (2002), "Determining the Number of Factors in Approximate Factor Models," Econometrica, $70(1), 191-221$.

[5] Baltagi, B. H., C. Kao, and F. Wang (2017), "Identification and Estimation of a Large Factor Model with Structural Instability", Journal of Econometrics, 197 (1), 87 - 100.

[6] Banbura, M. D., D. Giannone, and L. Reichlin (2011), "Nowcasting," in M. P. Clements and D. F. Hendry (eds.), The Handbook of Economic Forecasting, 193 - 224, Oxford: Oxford University Press.

[7] Barigozzi, M., M. Hallin, and S. Soccorsi (2019), "Identification of Global and Local Shocks in International Financial Markets via General Dynamic Factor Models", Journal of Financial Econometrics, $17(3), 462-494$.

[8] Bekaert, G., E. Engstrom, and Y. Xing (2009), "Risk, Uncertainty and Asset Prices," Journal of Financial Economics, $91(1), 59-82$.

[9] Boivin, J., and S. Ng (2006), “Are More Data Always Better for Factor Analysis?," Journal of Econometrics, $132(1), 169-194$.

[10] Bossaerts, P., and P. Hillion (1999), "Implementing Statistical Criteria to Select Return Forecasting Models: What Do We Learn?," Review of Financial Studies, 12 (2), 405 - 428.

[11] Chamberlain, G., and M. Rothschild (1983), "Arbitrage, Factor Structure, and Mean-Variance Analysis on Large Asset Markets," Econometrica, 51 (5), 1281 - 1304.

[12] Çakmakl, C., and D. van Dijk, (2016), "Getting the Most out of Macroeconomic Information for Predicting Excess Stock Returns", International Journal of Forecasting, 32(3), 650 - 668.

[13] Campbell, J. Y., and J. H. Cochrane (1999), "By Force or Habit: A Consumption-Based Explanation of Aggregate Stock Market Behavior," Journal of Political Economy, 107 (2), 205 - 251.

[14] Campbell, J. Y., and S. B. Thompson (2008), "Predicting Excess Stock Returns Out of Sample: Can Anything Beat the Historical Average?," Review of Financial Studies, 21 (4), 1509 - 1531.

[15] Clark, T. E., and K. D. West (2007), "Approximately Normal Tests for Equal Predictive Accuracy in Nested Models," Journal of Econometrics, 138 (1), $291-311$. 
[16] Clark, T. E., and M. W. McCracken (2009), "Improving Forecast Accuracy by Combining Recursive and Rolling Forecasts," International Economic Review, 50 (2), $363-395$.

[17] Connor, G., and R. A. Korajczyk (1986), "Performance Measurement with the Arbitrage Pricing Theory: A New Framework for Analysis," Journal of Financial Economics, 15 (3), 373 - 394.

[18] Connor, G., and R. A. Korajczyk (1988), "Risk and Return in an Equilibrium APT: Application of a New Test Methodology," Journal of Financial Economics, 21 (2), 255 - 289.

[19] Diebold, F. X. (2015), “Comparing Predictive Accuracy, Twenty Years Later: A Personal Perspective on the Use and Abuse of Diebold-Mariano Tests," Journal of Business 86 Economic Statistics, 33 (1), $1-24$.

[20] Farmer, L., Schmidt, L., and A., Timmermann, (2019), "Pockets of Predictability", SSRN working paper 3152386, dx.doi.org/10.2139/ssrn.3152386.

[21] Ferreira, M. A., and P. Santa-Clara (2011), "Forecasting Stock Market Returns: The Sum of the Parts Is More than the Whole," Journal of Financial Economics, 100 (3), 514 - 537.

[22] Fleming, J., C. Kirby, and B. Ostdiek (2001), "The Economic Value of Volatility Timing", Journal of Finance, $56(1), 329-353$.

[23] Forni, M., A. Giovannelli, M. Lippi, and S. Soccorsi (2018), "Dynamic Factor Model with InfiniteDimensional Factor Space: Forecasting," Journal of Applied Econometrics, 33 (5), 625 - 642.

[24] Forni, M., M. Hallin, M. Lippi, and L. Reichlin (2000), "The Generalized Dynamic Factor Model: Identification and Estimation," Review of Economics and Statistics, 82 (4), 540 - 554 .

[25] Forni, M., M. Hallin, M. Lippi, and L. Reichlin (2005), "The Generalized Dynamic Factor Model: One Sided Estimation and Forecasting," Journal of the American Statistical Association, 100 (471), $830-840$.

[26] Forni, M., M. Hallin, M. Lippi, and P. Zaffaroni (2015), "Dynamic Factor Models with InfiniteDimensional Factor Spaces: One-Sided Representations," Journal of Econometrics, 185 (2), 359 371. 
[27] Forni, M., M. Hallin, M. Lippi, and P. Zaffaroni (2017), "Dynamic Factor Models with InfiniteDimensional Factor Space: Asymptotic analysis," Journal of Econometrics, 199(1), 74 - 92.

[28] Gargano, A., D. Pettenuzzo, and A. Timmermann (2017), "Bond Return Predictability: Economic Value and Links to the Macroeconomy," Management Science, https://doi.org/10.1287/mnsc.2017.2829.

[29] Geweke, J. (1977), "The Dynamic Factor Analysis of Economic Time Series," in D. J. Aigner and A. S. Goldberger (eds.), Latent Variables in Socio-Economic Models, North-Holland.

[30] Ghysels, E., Horan, C., Moench, E. (2018), "Forecasting through the Rearview Mirror: Data Revisions and Bond Return Predictability", Review of Financial Studies, 31, 678-714.

[31] Giacomini, R., and Rossi, B. (2010). "Forecast comparisons in unstable environments." Journal of Applied Econometrics, 25(4), $595-620$.

[32] Giacomini, R., and H. White (2006), "Tests of Conditional Predictive Ability," Econometrica, 74 (6), $1545-1578$.

[33] Giannone, D., M. Lenza, and G. E. Primiceri (2017), "Economic Predictions with Big Data: The Illusion of Sparsity," CEPR Discussion Paper No. DP12256.

[34] Gonçalves, S., M. W. McCracken, and B. Perron (2017), "Tests of Equal Accuracy for Nested Models with Estimated Factors", Journal of Econometrics, 198(2), 231 - 252.

[35] Goyal, A., and I. Welch (2003), "Predicting the Equity Premium with Dividend Ratios," Management Science, 49 (5), $639-654$.

[36] Granger, C. W. J., and M. H. Pesaran (2000), "Economic and Statistical Measures of Forecast Accuracy," Journal of Forecasting, 19 (7), 426 - 459.

[37] Hallin, M., and M. Lippi (2013). "Factor Models in High-Dimensional Time Series - A Time-Domain Approach", Stochastic Processes and their Applications, 123(7), 2678 - 2695.

[38] Hallin, M., and R. Liška (2007), "Determining the Number of Factors in the General Dynamic Factor Model," Journal of the American Statistical Association, 102 (48), 603 - 617. 
[39] Hansen, P. R., and A. Timmermann (2012), "Choice of Sample Split in Out-of-Sample Forecast Evaluation," Working Paper, European University Institute and University of California, San Diego.

[40] Henkel, S. J., J. S. Martin, and F. Nardari (2011), "Time-Varying Short-Horizon Predictability," Journal of Financial Economics, 99 (3), 560 - 580.

[41] Inoue, A., L. Jin, and B. Rossi (2017), "Rolling Window Selection for Out-of-Sample Forecasting with Time-Varying Parameters," Journal of Econometrics, 196 (1), 55 - 67.

[42] Leicht, G., and J. E. Tanner (1991), "Economic Forecast Evaluation: Profits Versus the Conventional Error Measures", American Economic Review, 81 (3), 580 - 590.

[43] Ludvigson, S. C., and S. Ng (2009), "The Empirical Risk-Return Relation: A Factor Analysis Approach", Journal of Financial Economics , 83(1), 171 - 222.

[44] Massacci, D. (2015), "Predicting the Distribution of Stock Returns: Model Formulation, Statistical Evaluation, VaR Analysis and Economic Significance," Journal of Forecasting, 34(3), 191 - 208.

[45] Menzly, L., T. Santos, and P. Veronesi (2004), "Understanding Predictability," Journal of Political Economy, $112(1), 1-47$.

[46] McCracken, M. W., and S. Ng (2016), "FRED-MD: A Monthly Database for Macroeconomic Research," Journal of Business 63 Economics Statistics, 34(4), 574 - 589.

[47] Neely, C. J., D. E. Rapach, J. Tu, and G. Zhou (2014), "Forecasting the Equity Risk Premium: The Role of Technical Indicators," Management Science, 60 (7), 1772-1791.

[48] Ohno, S., and T. Ando (2018), "Stock Return Predictability: A Factor-Augmented Predictive Regression System with Shrinkage Method," Econometric Reviews, 37(1), 29 - 60.

[49] Pan, Z., D. Pettenuzzo, and Y. Wang (2020) "Forecasting Stock Returns: A Predictor-Constrained Approach," Journal of Empirical Finance, 55, $200-217$.

[50] Paye, B. S., and A. Timmermann (2006), "Instability of Return Prediction Models," Journal of Empirical Finance, $13(3), 274-315$.

[51] Pesaran, M. H., and A. Timmermann (1995), "Predictability of Stock Returns: Robustness and Economic Significance," Journal of Finance, 50 (4), 1201 - 1228. 
[52] Pesaran, M. H., and A. Timmermann (2005), "Real-Time Econometrics," Econometric Theory, $21(1), 212-231$.

[53] Pesaran , M. H., and A. Timmermann (2007), "Selection of Estimation Window in the Presence of Breaks", Journal of Econometrics, 137(1) , 134-61.

[54] Pettenuzzo, D., A. Timmermann, and R. Valkanov (2014), "Forecasting Stock Returns under Economic Constraints," Journal of Financial Economics, $114(3), 517$ - 553.

[55] Rapach, D. E., J. K. Strauss, and G. Zhou (2010), "Out-of-Sample Equity Premium Prediction: Combination Forecasts and Links to the Real Economy," Review of Financial Studies, 23 (2), 821 862.

[56] Rapach, D. E., and M. E. Wohar (2006), "Structural Breaks and Predictive Regression Models of Aggregate U.S. Stock Returns,” Journal of Financial Econometrics, 4 (2), 238 - 274 .

[57] Rapach, D. E., and G. Zhou (2013), "Forecasting Stock Returns," in G. Elliott and A. Timmermann (eds.), Handbook of Economic Forecasting, Volume 2, Part A, 329 - 383, Amsterdam: NorthHolland.

[58] Sargent T., and C. A. Sims (1977), "Business Cycle Modelling without Pretending to Have too Much a Priori Economic Theory," in C. A. Sims (eds.), New Methods in Business Research, Federal Reserve Bank of Minneapolis.

[59] Soccorsi, S. (2016), "Measuring Nonfundamentalness for Structural VARs", Journal of Economic Dynamics and Control, 71, $86-101$.

[60] Stock, J. H., and M. W. Watson (2002a), "Forecasting Using Principal Components from a Large Number of Predictors," Journal of the American Statistical Association, 97 (460), 1167 - 1179.

[61] Stock, J. H., and M. W. Watson (2002b). "Macroeconomic Forecasting Using Diffusion Indexes", Journal of Business \& Economic Statistics , 20(2), 147 - 162.

[62] Stock, J. H., and M. W. Watson (2012), "Generalized Shrinkage Methods for Forecasting Using Many Predictors," Journal of Business 83 Economic Statistics, 30 (4), 481 - 493. 
[63] Timmermann, A. (2008), "Elusive Return Predictability," International Journal of Forecasting, $24(1), 1-18$.

[64] Welch, I., and A. Goyal (2008), "A Comprehensive Look at the Empirical Performance of Equity Premium Prediction," Review of Financial Studies, 21 (4), 1455 - 1508. 
Table 1: Summary Statistics, 1960 - 2014

Notes. This table reports summary statistics for excess returns, calculated as continuously compounded returns on the S\&P 500 index in excess of the treasury bill rate, and for the following 14 predictors proposed in Welch and Goyal (2008): log dividend-price ratio (log (DP)), log dividend-yield $(\log (\mathrm{DY}))$, log earnings-price ratio (log (EP)), log dividend-payout ratio $(\log (\mathrm{DE}))$, stock variance (SVAR), book-to-market ratio (BM), net equity expansion (NTIS), treasury bill rate (TBL), long-term yield (LTY), long-term return (LTR), term spread (TMS), default yield spread (DFY), default return spread (DFR), inflation (INFL). The sample period is $1960-2014$.

\begin{tabular}{lcccc}
\hline \hline Variables & Mean & Std. Deviation & Skewness & Kurtosis \\
\hline Excess Returns & 0.001 & 0.043 & -0.693 & 5.447 \\
$\log ($ DP) & -3.577 & 0.397 & -0.305 & 2.350 \\
$\log (\mathrm{DY})$ & -3.571 & 0.397 & -0.312 & 2.379 \\
$\log (\mathrm{EP})$ & -2.829 & 0.432 & -0.760 & 6.120 \\
$\log (\mathrm{DE})$ & -0.748 & 0.317 & 2.667 & 17.390 \\
SVAR & 0.002 & 0.004 & 10.162 & 136.180 \\
BM & 0.509 & 0.259 & 0.690 & 2.646 \\
NTIS & 0.012 & 0.019 & -0.883 & 3.984 \\
TBL & 0.049 & 0.031 & 0.698 & 4.036 \\
LTY & 0.067 & 0.026 & 0.804 & 3.264 \\
LTR & 0.006 & 0.029 & 0.425 & 5.827 \\
TMS & 0.018 & 0.015 & -0.289 & 2.732 \\
DFY & 0.010 & 0.005 & 1.739 & 7.156 \\
DFR & 0.000 & 0.014 & -0.366 & 9.763 \\
INFL & 0.003 & 0.003 & 0.008 & 6.693 \\
\hline
\end{tabular}




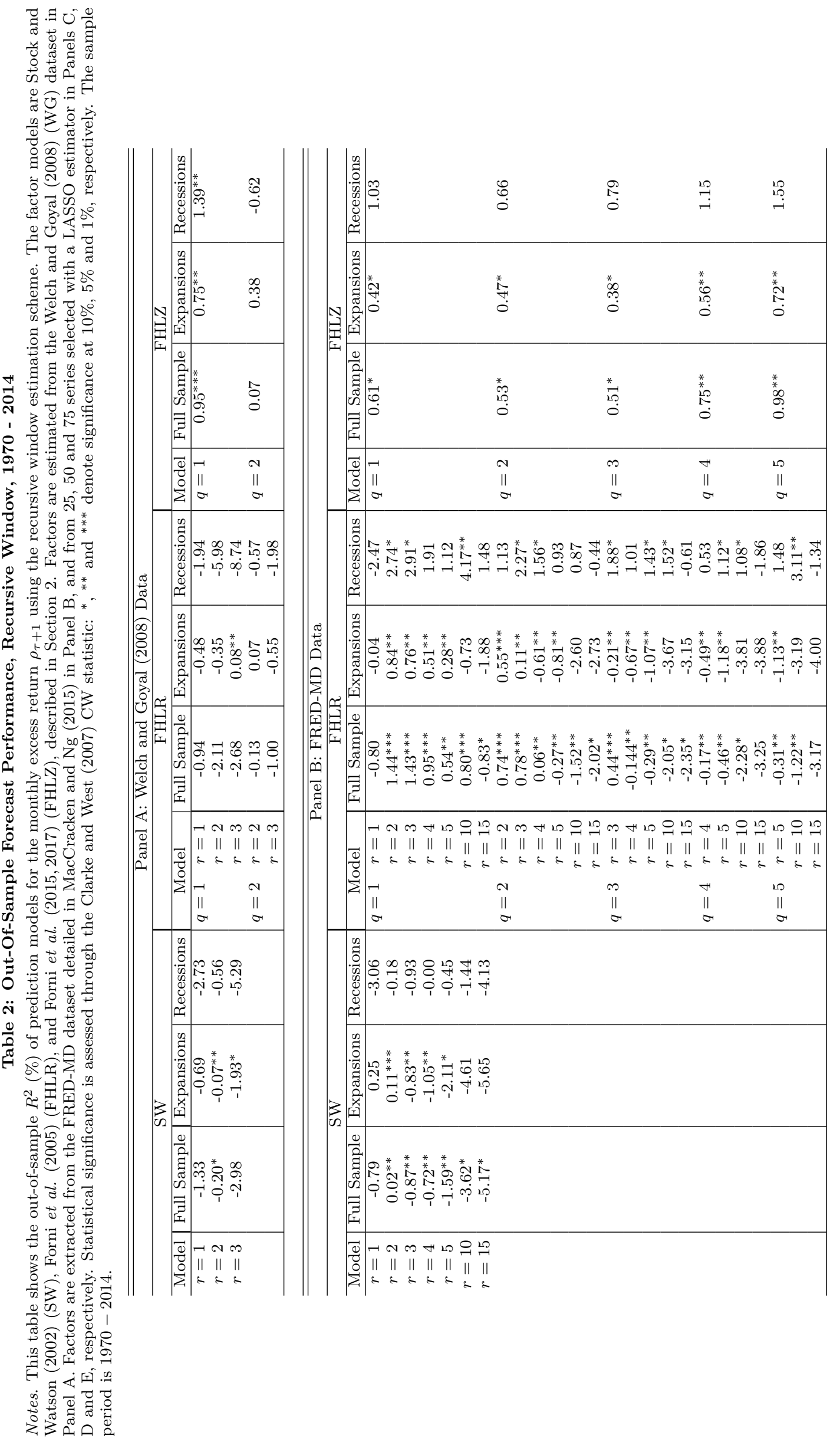




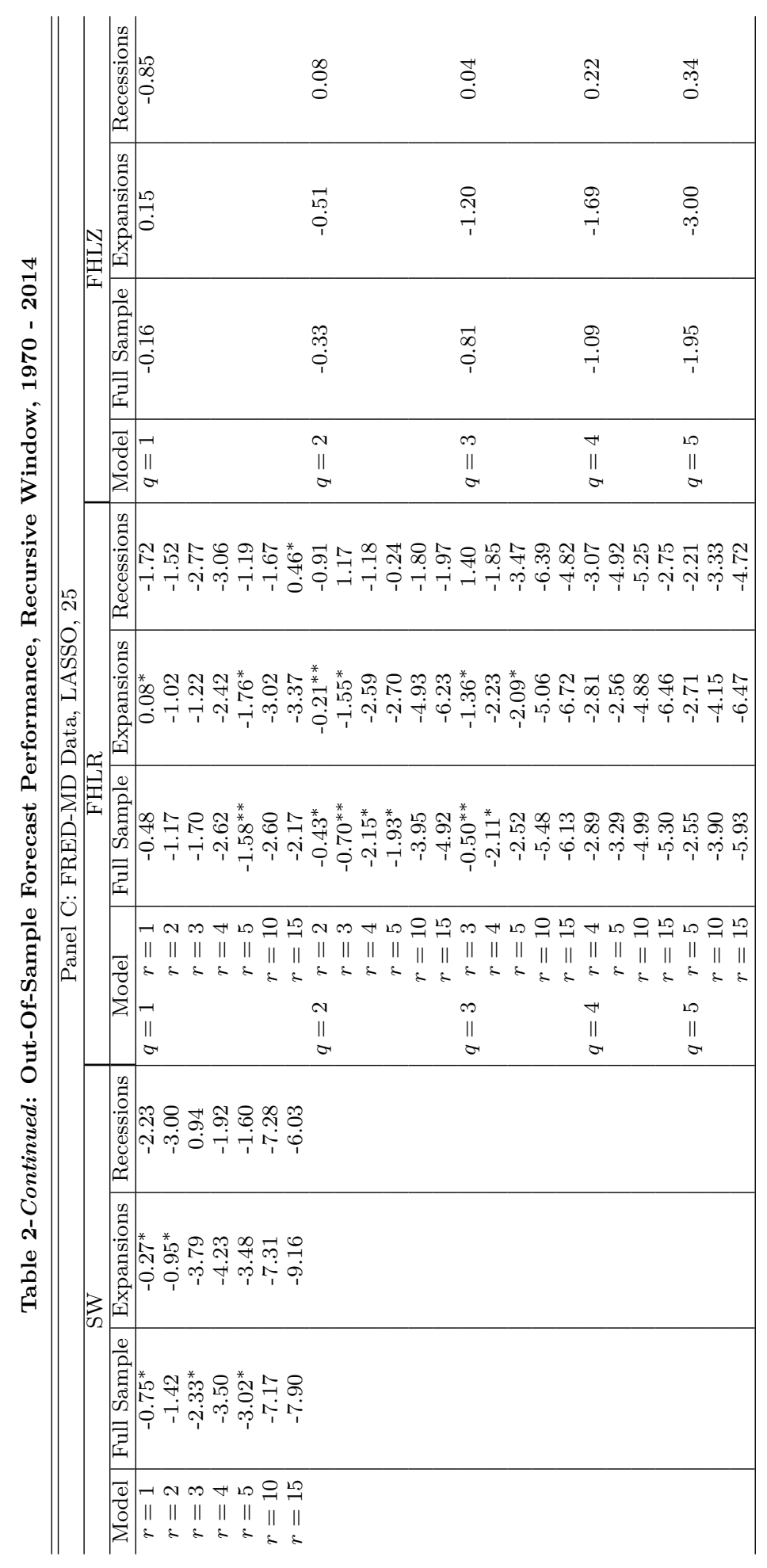




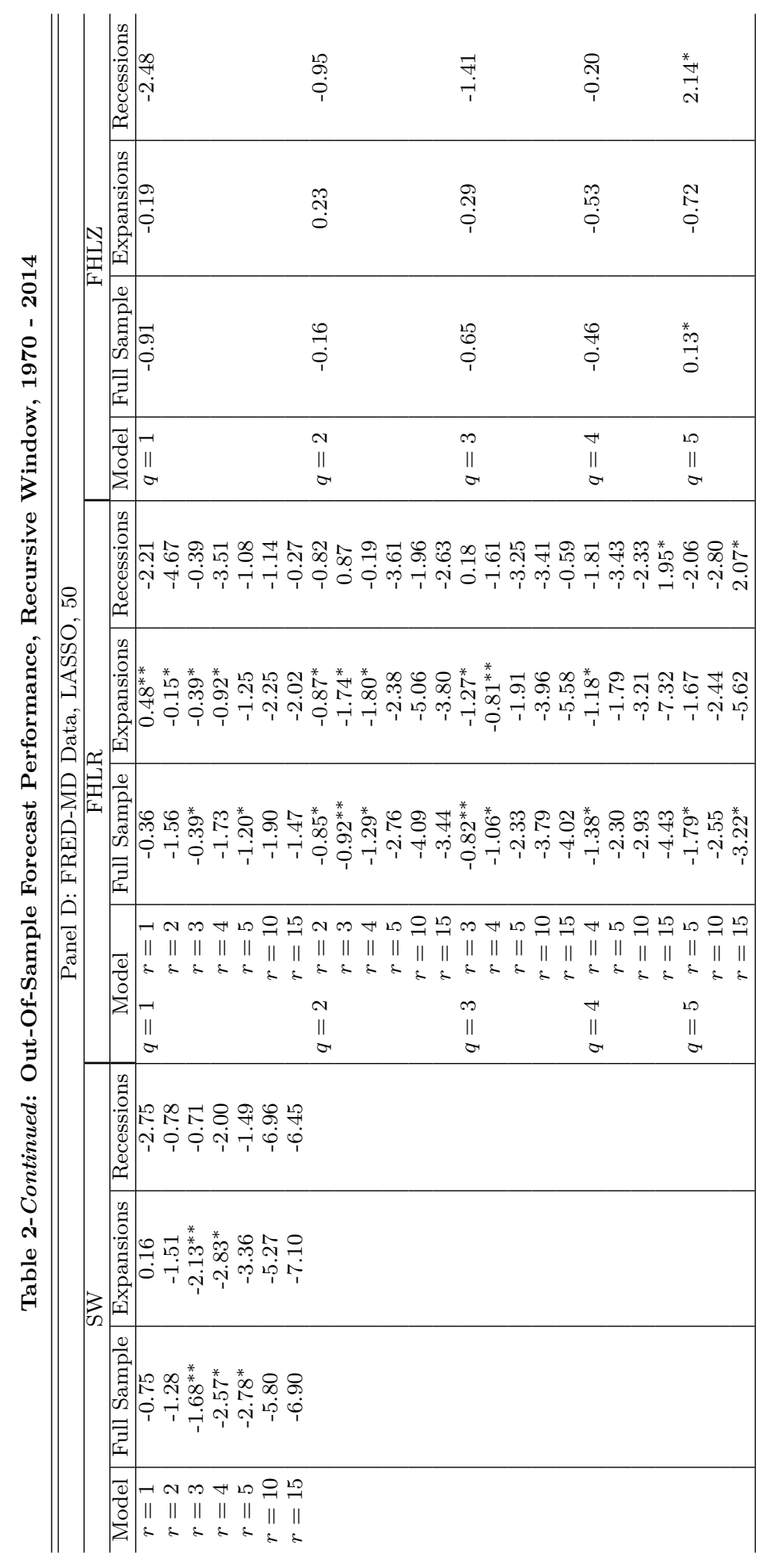




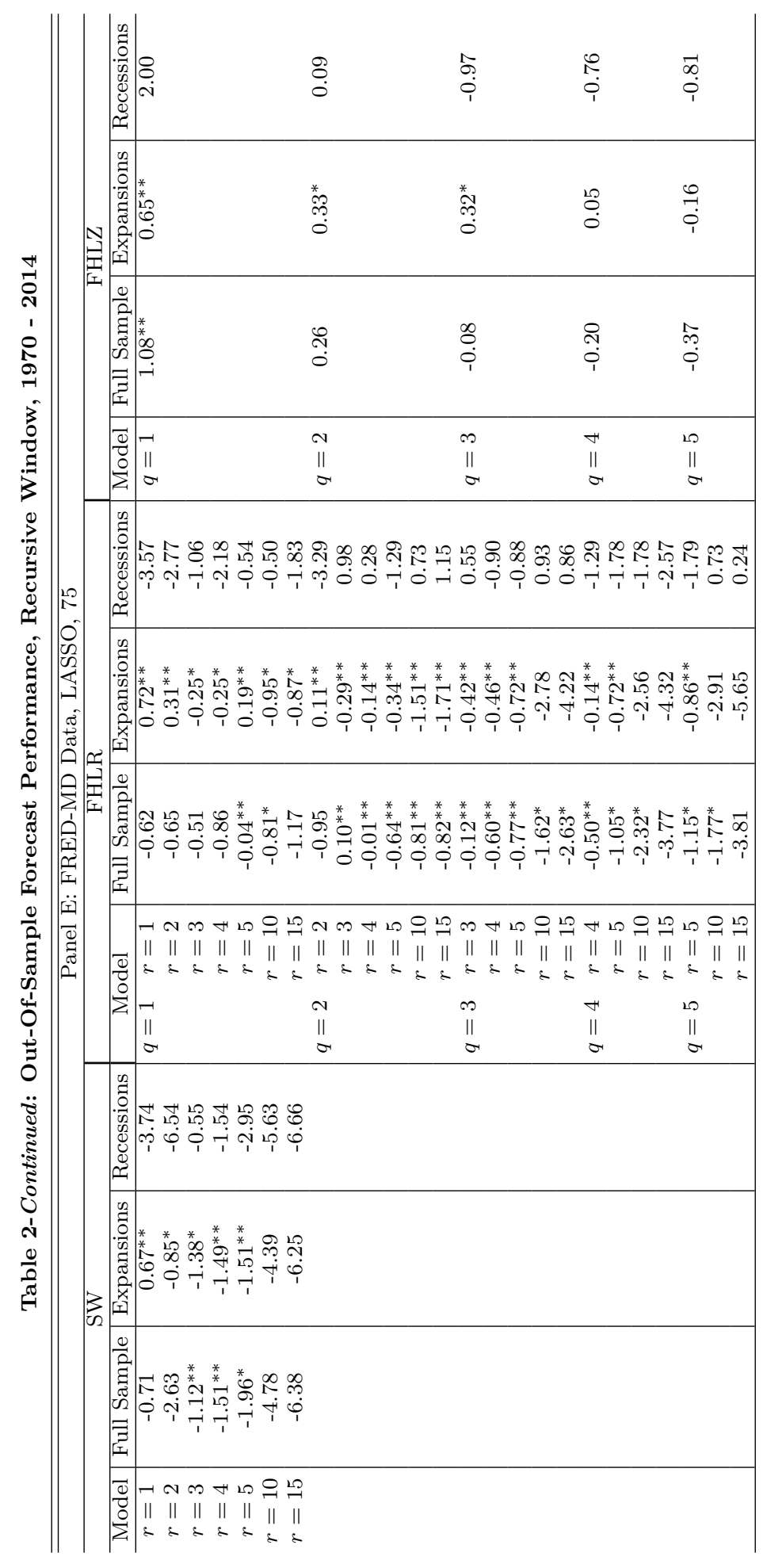




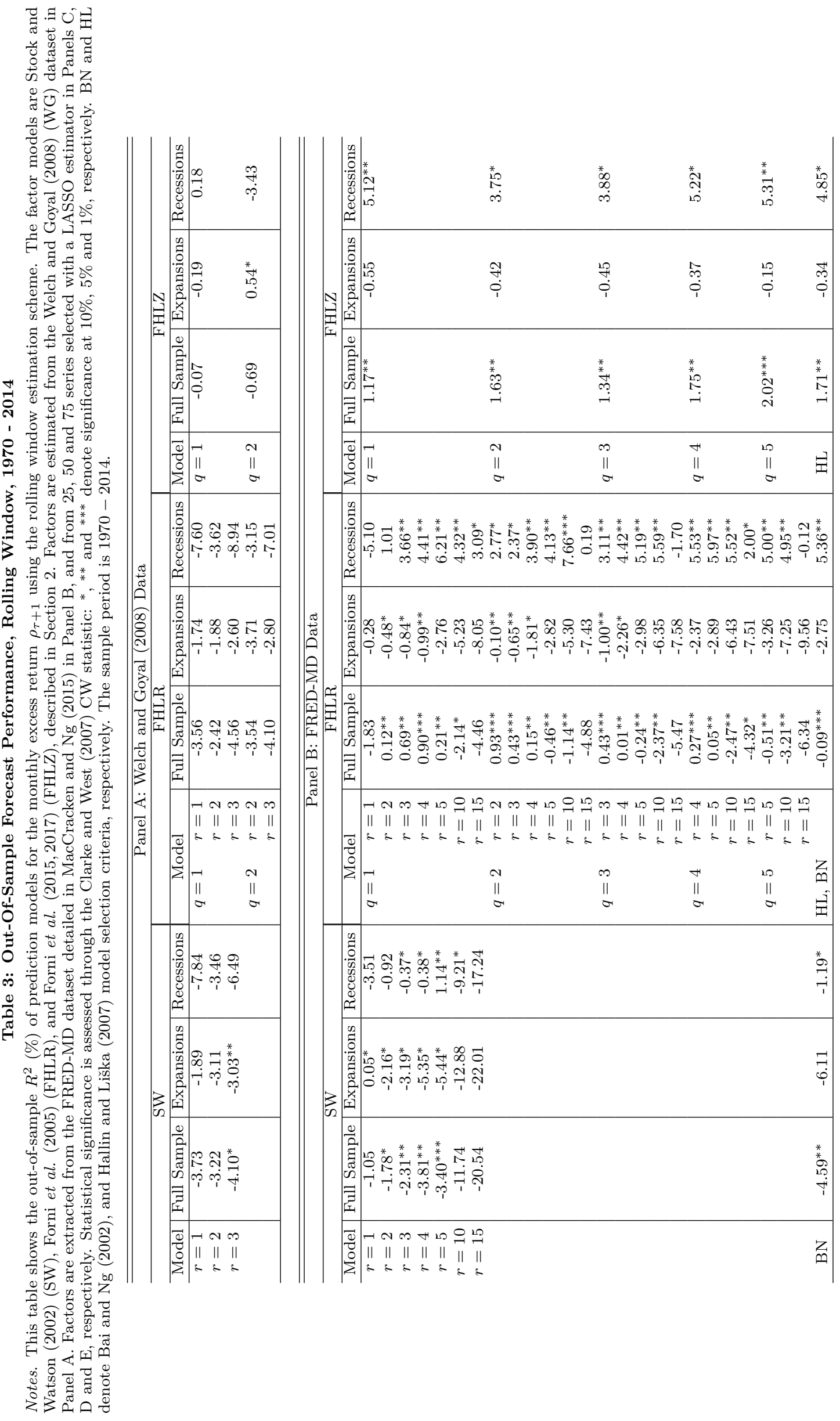




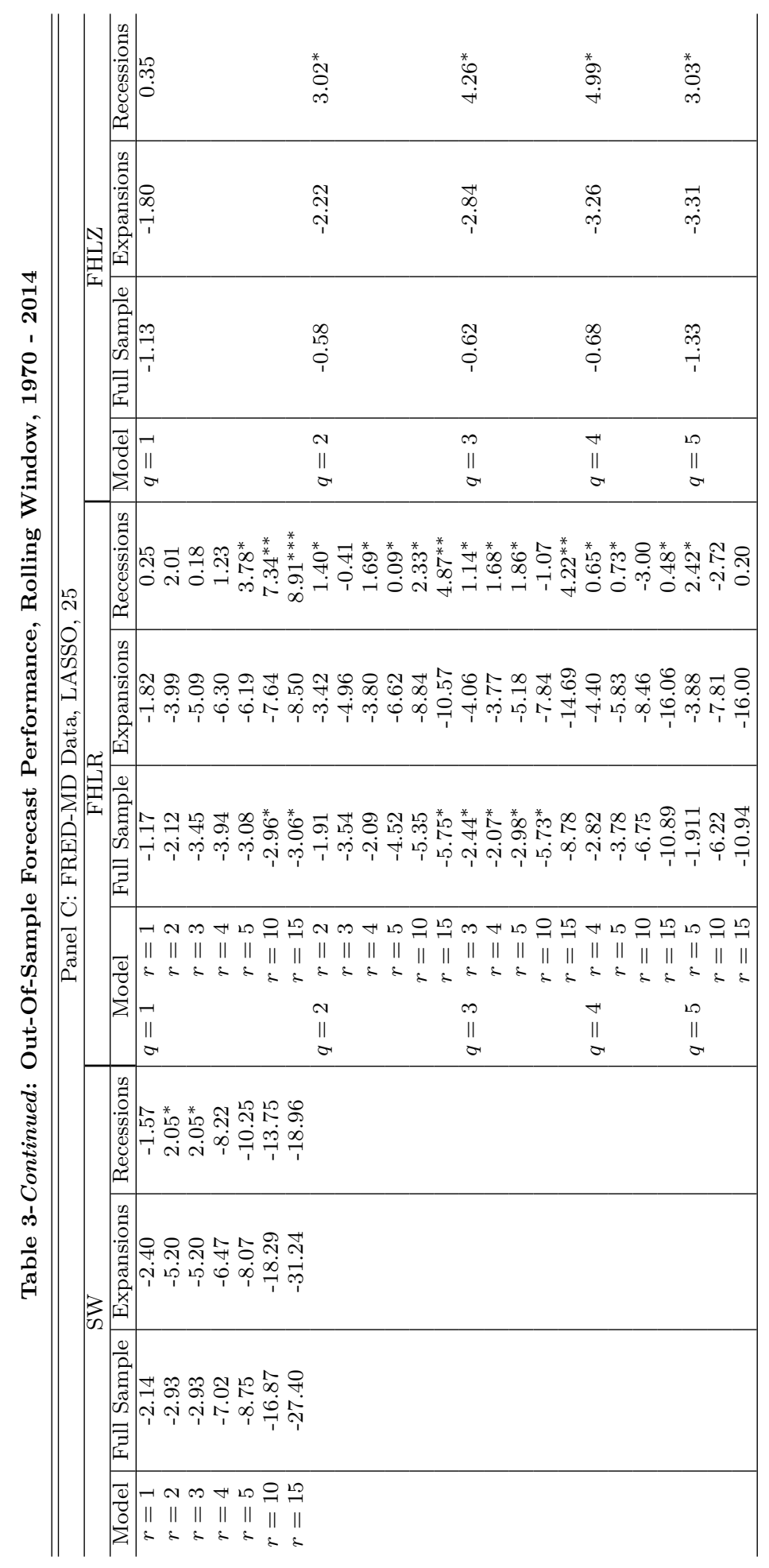




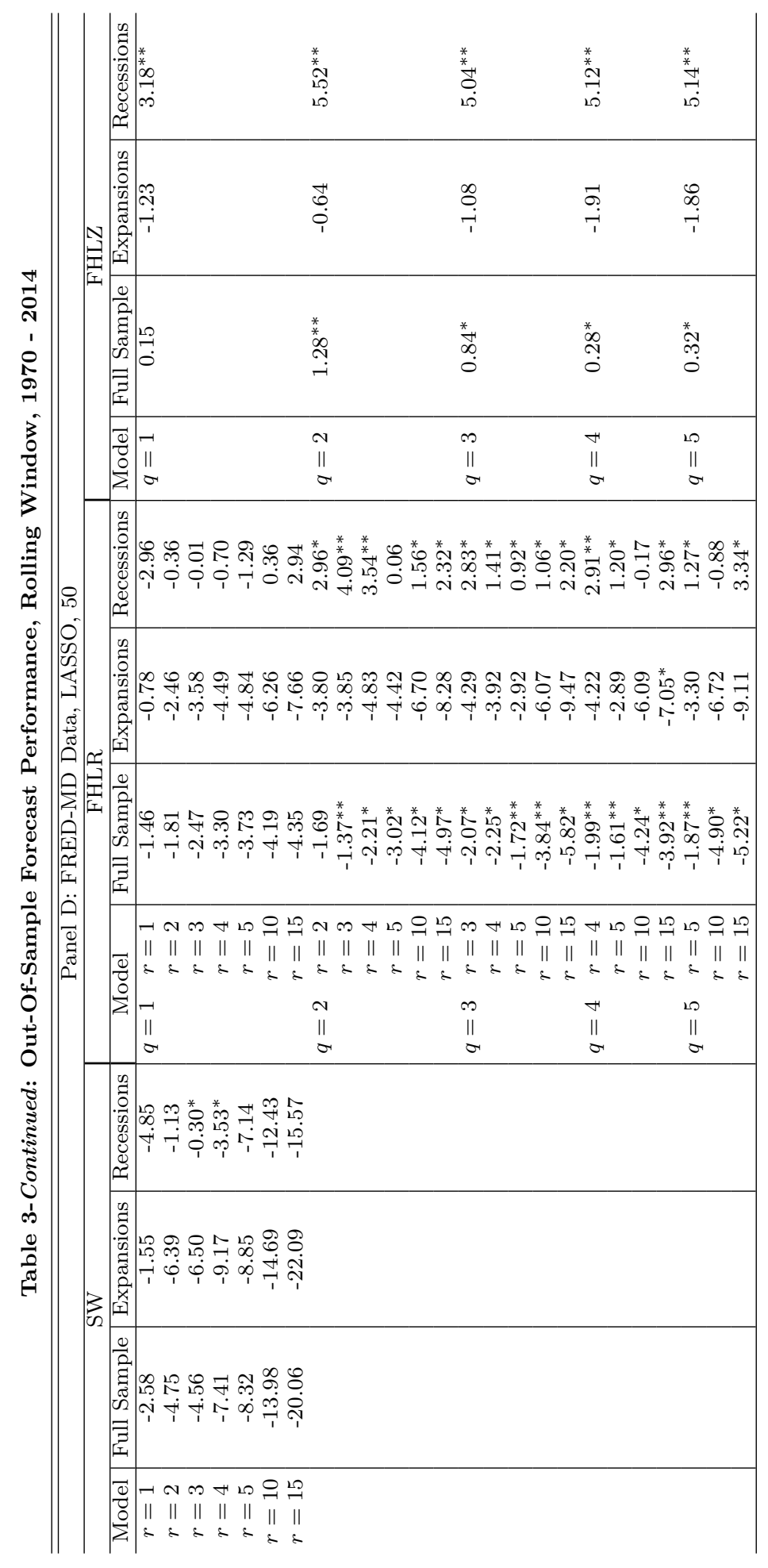




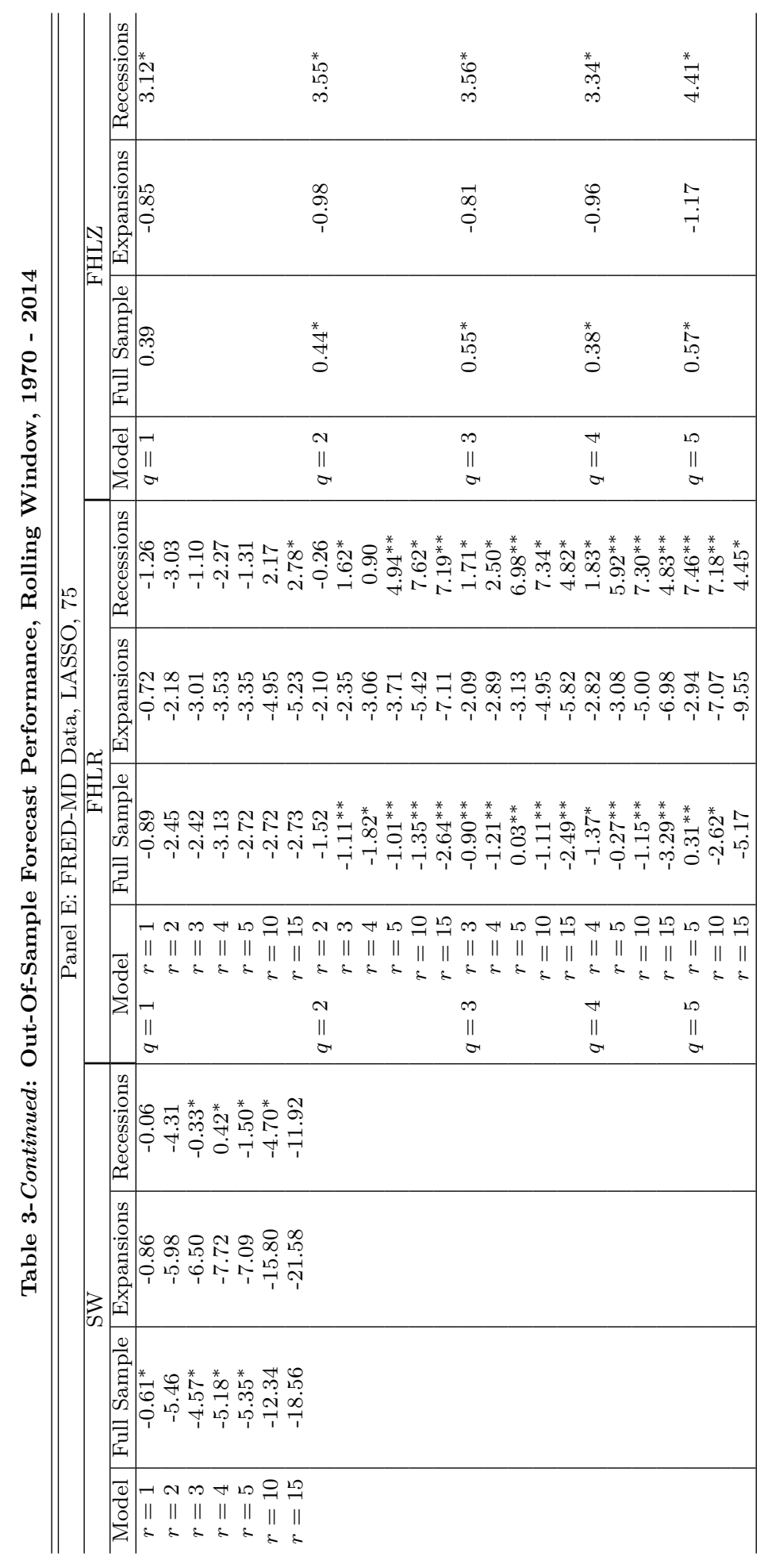


Table 4: Resume of small to medium LASSO Results, FRED-MD Data, 1970 - 2014

Notes. This table shows the out-of-sample $R^{2}(\%)$ of prediction models for the monthly excess return $\rho_{\tau+1}$ using recursive window (Panel A) and rolling window (Panel B) estimation schemes. The factor models are Stock and Watson (2002) (SW), Forni et al. (2005) (FHLR), and Forni et al. (2015, 2017) (FHLZ), described in Section 2 . Factors are extracted from 25, 50 and 75 series selected at each point in time with a LASSO estimator applied to the FRED-MD dataset detailed in MacCracken and $\mathrm{Ng}$ (2015). The sample period is $1970-2014$.

\begin{tabular}{lrrrrr}
\hline \hline & & LASSO, 25 & LASSO, 50 & LASSO, 75 & full FRED-MD dataset \\
\hline \multirow{3}{*}{ Panel A: Recursive Window } & SW & -0.75 & -0.75 & -0.71 & 0.02 \\
& FHLR & -0.16 & -0.36 & 0.10 & 1.44 \\
& FHLZ & -0.50 & 0.13 & 1.08 & 0.98 \\
\hline \multirow{2}{*}{ Panel B: Rolling Window } & SW & -2.14 & -2.58 & -0.61 & -1.05 \\
& FHLR & -1.17 & -1.37 & 0.31 & 0.93 \\
& FHLZ & -0.58 & 1.28 & 0.57 & 2.02 \\
\hline
\end{tabular}




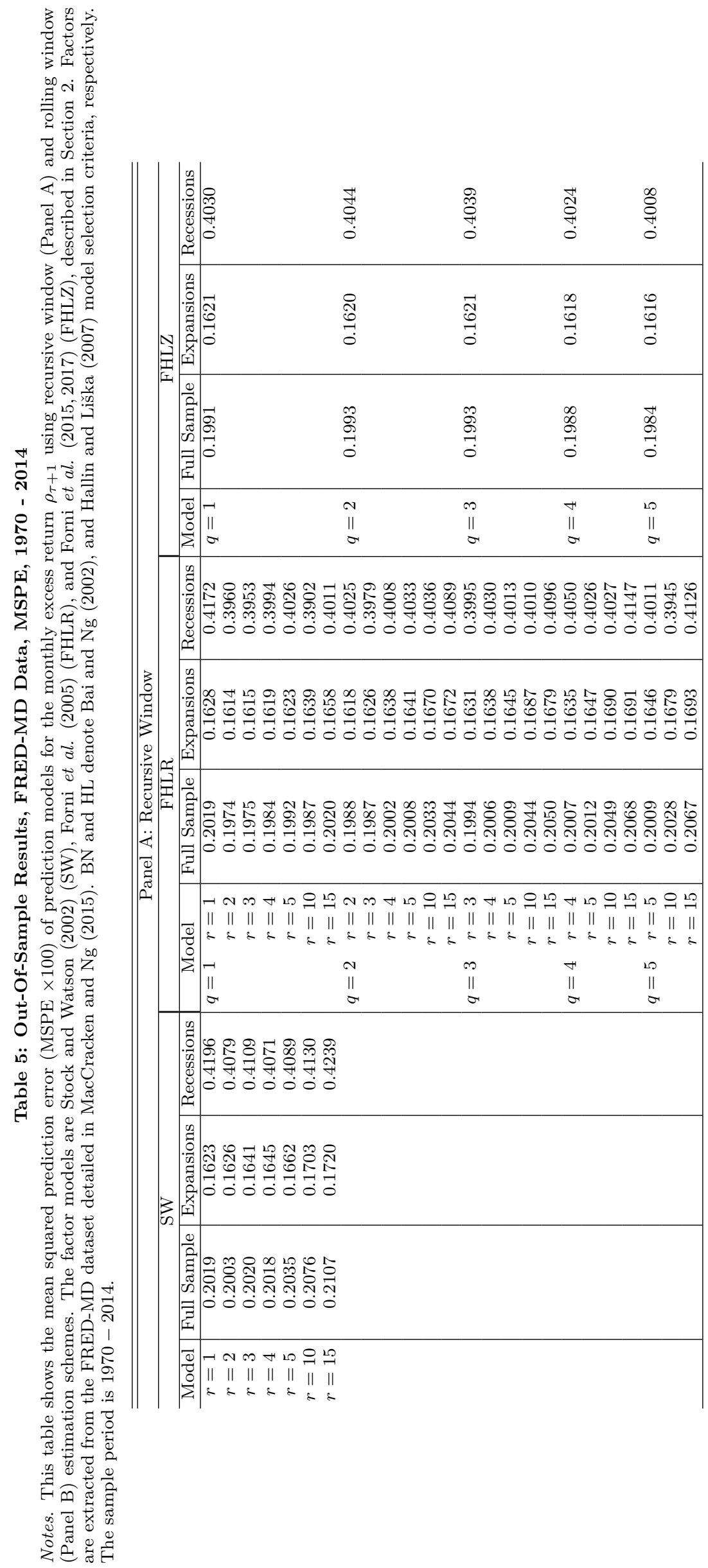




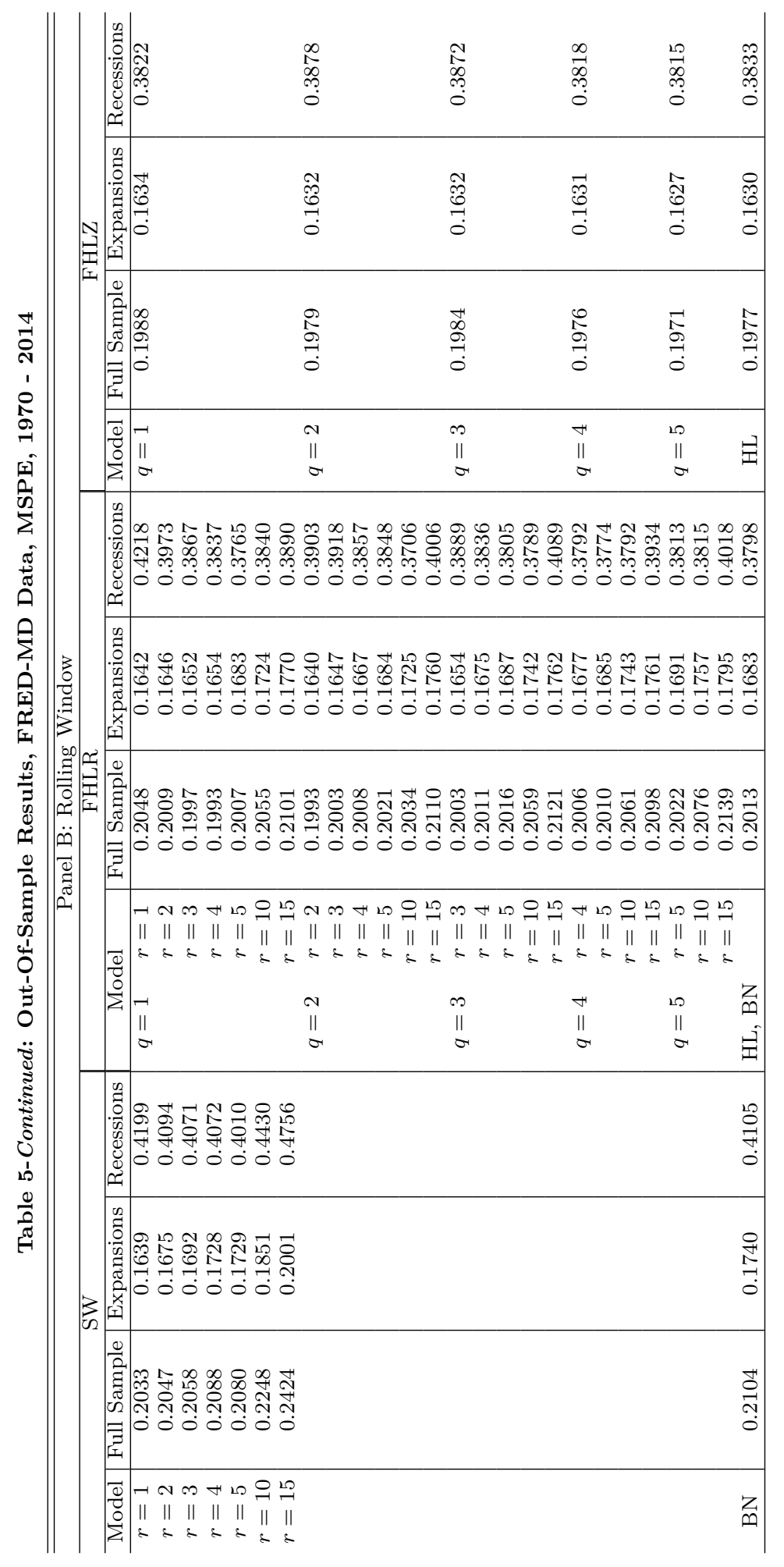




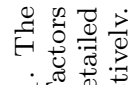

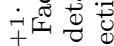

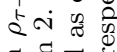

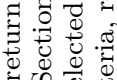

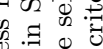

X广仓

$>0$

5 o :

G

年

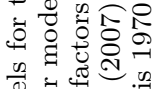

$+\frac{5}{0}=0$

穴

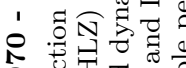

战 :

ป

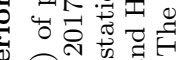
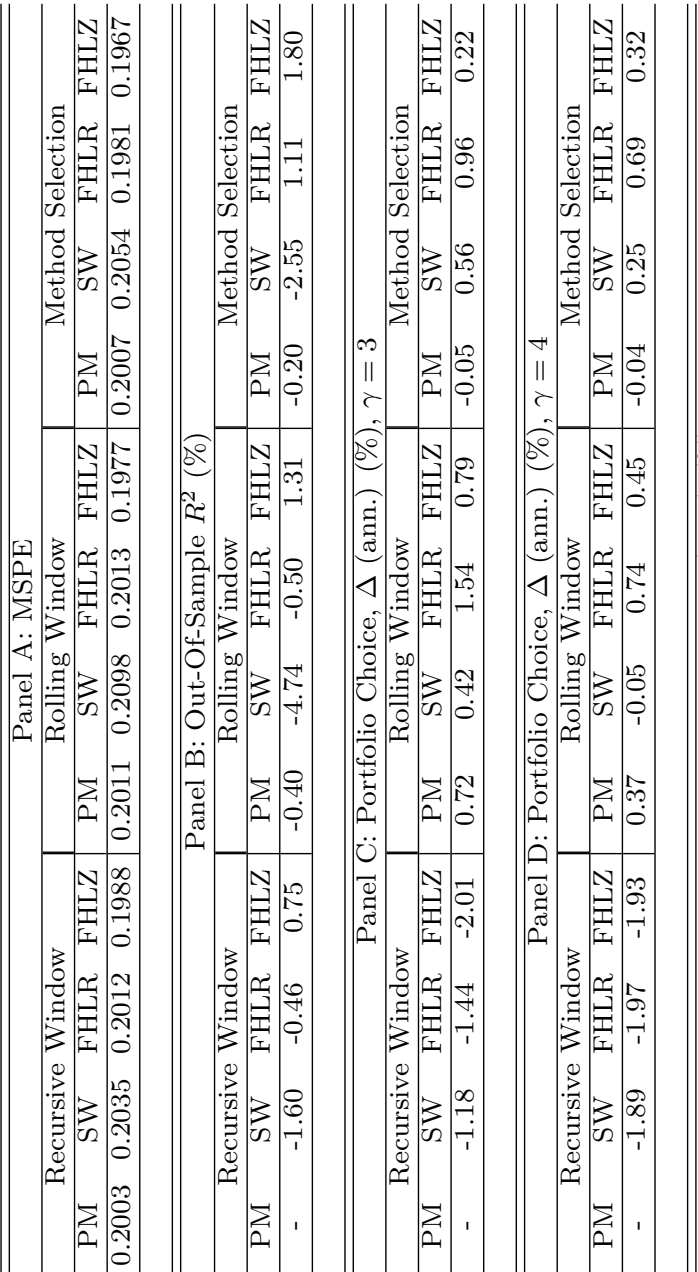

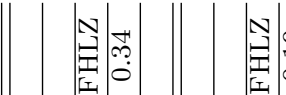

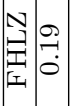

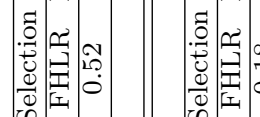

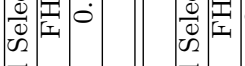

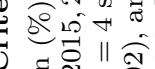

: \% व

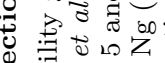

क $\vec{\exists} \| \vec{g}$

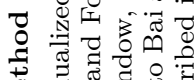

$\sum_{1}^{0}$ का

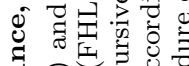

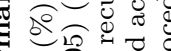

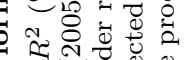

ब.

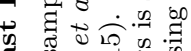

i $z 50$

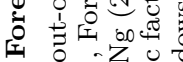

응

的

语

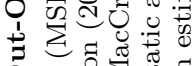

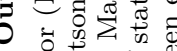

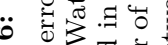

을

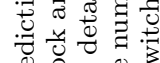

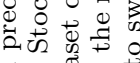

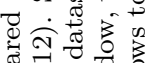

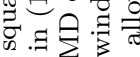

สี ซื่

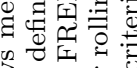

. 0 o 0

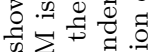

o

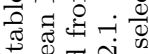

on 의

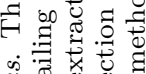

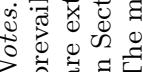

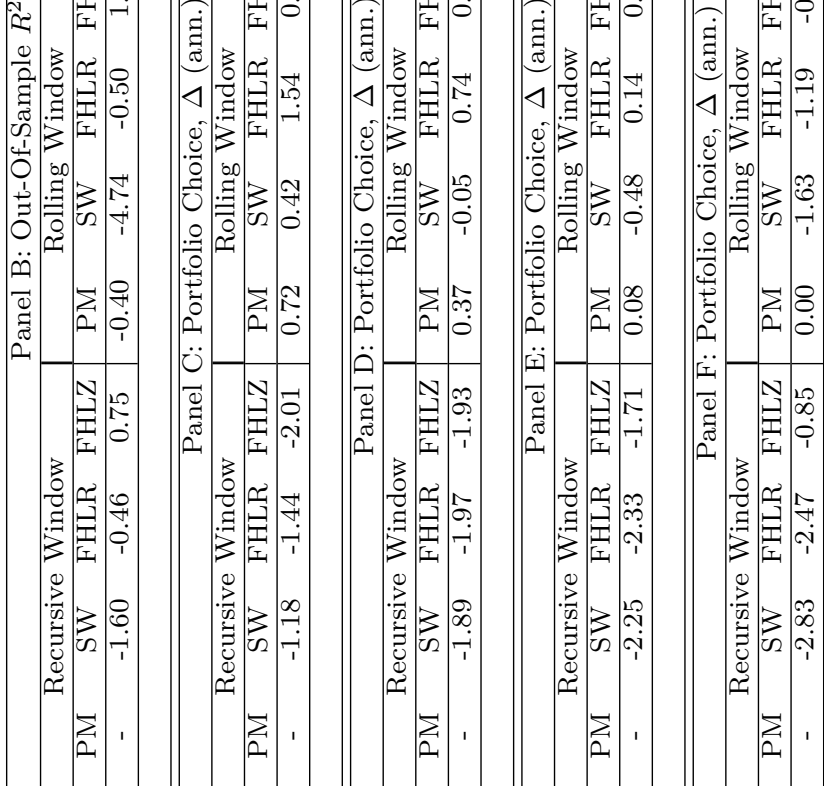


Table 7: Real-time vs pseudo real-time results

Notes. This table shows mean squared prediction error (MSPE $\times 100)$ and out-of-sample $R^{2}(\%)$ of prediction models for the monthly excess return $\rho_{\tau+1}$. The prevailing mean PM is defined in (12). Stock and Watson (2002a) (SW), Forni et al. (2005) (FHLR), and Forni et al. (2015, 2017) (FHLZ) factor models are described in Section 2 . Factors are extracted from the FRED-MD dataset detailed in MacCracken and $\mathrm{Ng}$ (2015). Under recursive window, $r=5$ and $q=4$ static and dynamic factors, respectively, are selected as detailed in Section 5.2.1. Under rolling window, the number of static and dynamic factors is selected according to Bai and $\mathrm{Ng}$ (2002), and Hallin and Liška (2007) model selection criteria, respectively. The method selection criterion allows to switch between estimation windows using the procedure described in Section 5.3.2. The sample period is indicated in the Table.

PSEUDO REAL-TIME

\begin{tabular}{|c|c|c|c|c|c|c|c|c|c|c|}
\hline \multicolumn{11}{|c|}{ PANEL A : MSPE based on FRED Dataset } \\
\hline \multicolumn{3}{|c|}{ Recursive Window (1992-2014) } & \multicolumn{4}{|c|}{ Rolling Window (1992-2014) } & \multicolumn{4}{|c|}{ Method Selection (2009-2014) } \\
\hline $\mathrm{PM}$ & SW & FHLR & FHLZ | & SW & FHLR & FHLZ | & $\mathrm{PM}$ & SW & FHLR & FHLZ \\
\hline 0.1772 & 0.1803 & 0.1785 & $0.1764 \quad 0.1787$ & 0.1925 & 0.1838 & 0.1771 & 0.1901 & 0.2141 & 0.1999 & 0.1996 \\
\hline \multicolumn{11}{|c|}{ PANEL B: Out-of-sample $R^{2}$ based on FRED Dataset } \\
\hline \multicolumn{3}{|c|}{ Recursive Window (1992-2014) } & \multicolumn{4}{|c|}{ Rolling Window (1992-2014) } & \multicolumn{4}{|c|}{ Method Selection (2009-2014) } \\
\hline $\mathrm{PM}$ & SW & FHLR & FHLZ & SW & FHLR & FHLZ | & $\mathrm{PM}$ & SW & FHLR & FHLZ \\
\hline- & -1.7155 & -0.7234 & $0.4970 \quad-0.8084$ & -8.5957 & -3.7107 & 0.0764 & -1.4267 & -14.2244 & -6.6276 & -6.4718 \\
\hline
\end{tabular}

REAL-TIME

\begin{tabular}{|c|c|c|c|c|c|c|c|c|c|c|}
\hline \multicolumn{11}{|c|}{ PANEL C : MSPE based on (ALFRED + ADS vintages) } \\
\hline \multicolumn{3}{|c|}{ Recursive Window (1992-2014) } & \multicolumn{4}{|c|}{ Rolling Window (1992-2014) } & \multicolumn{4}{|c|}{ Method Selection (2009-2014) } \\
\hline $\mathrm{PM}$ & SW & FHLR & $\mathrm{PM}$ & SW & FHLR & FHLZ & $\mathrm{PM}$ & SW & FHLR & FHLZ \\
\hline 0.1772 & 0.1793 & 0.1775 & 0.1787 & 0.1796 & 0.1802 & 0.1728 & 0.1901 & 0.1919 & 0.1894 & 0.189 \\
\hline \multicolumn{11}{|c|}{ PANEL D: Out-of-sample $R^{2}$ based on (ALFRED + ADS vintages) } \\
\hline \multicolumn{3}{|c|}{ Recursive Window (1992-2014) } & \multicolumn{4}{|c|}{ Rolling Window (1992-2014) } & \multicolumn{4}{|c|}{ Method Selection (2009-2014) } \\
\hline $\mathrm{PM}$ & SW & FHLR & $\mathrm{PM}$ & SW & FHLR & FHLZ & $\mathrm{PM}$ & SW & FHLR & FHLZ \\
\hline- & -1.1303 & -0.1666 & -0.8084 & -1.3370 & -1.6882 & $2.5080 * *$ & -1.4242 & -2.3649 & -1.0163 & -0.8175 \\
\hline
\end{tabular}


Figure 1: Degree of commonality

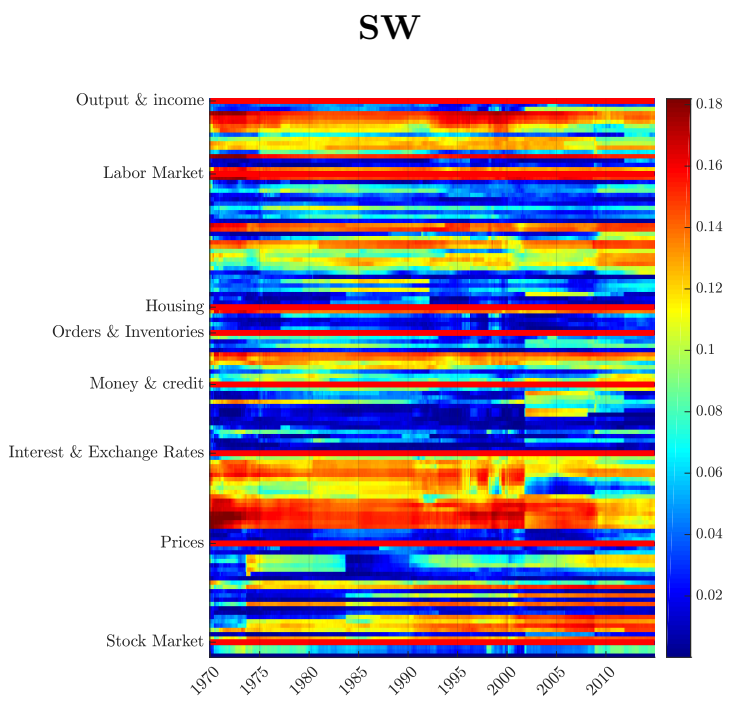

FHLR

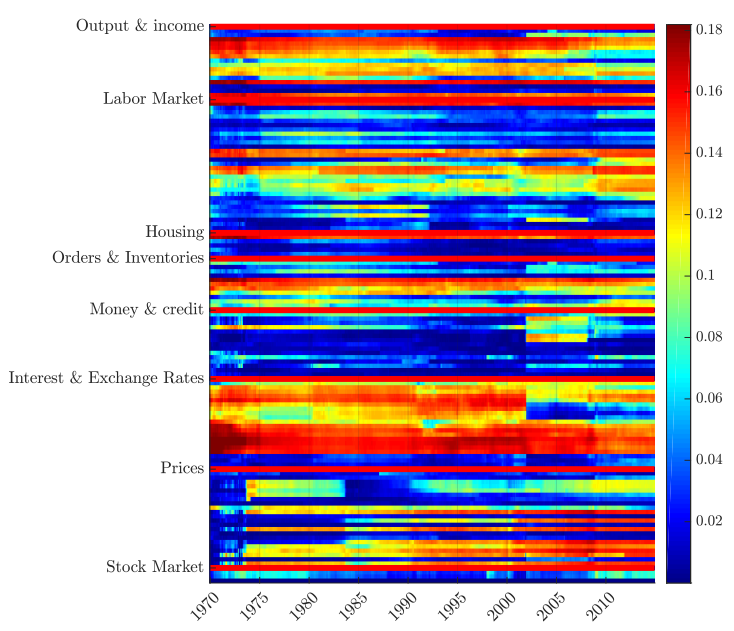

FHLZ

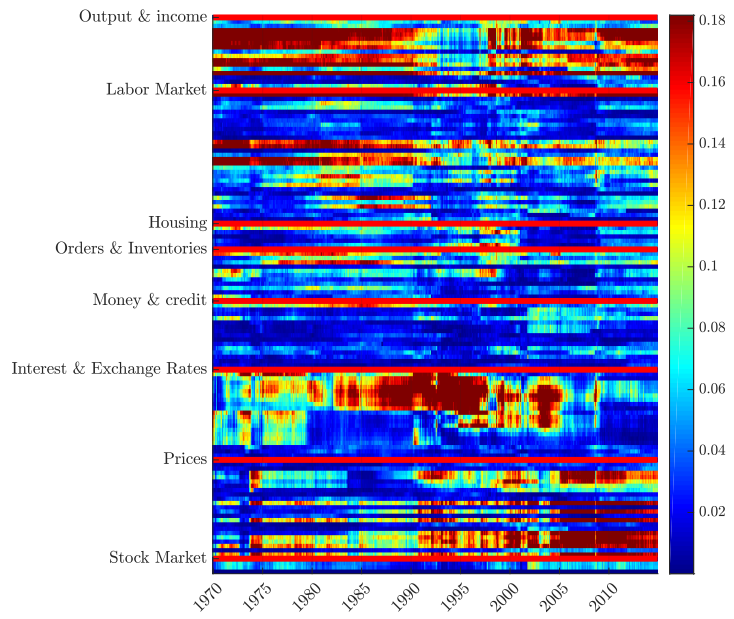

Notes. The heatmaps above report the (rolling) degree of commonality estimated by the three factor models as in equation (11) 


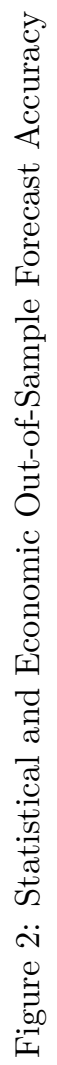
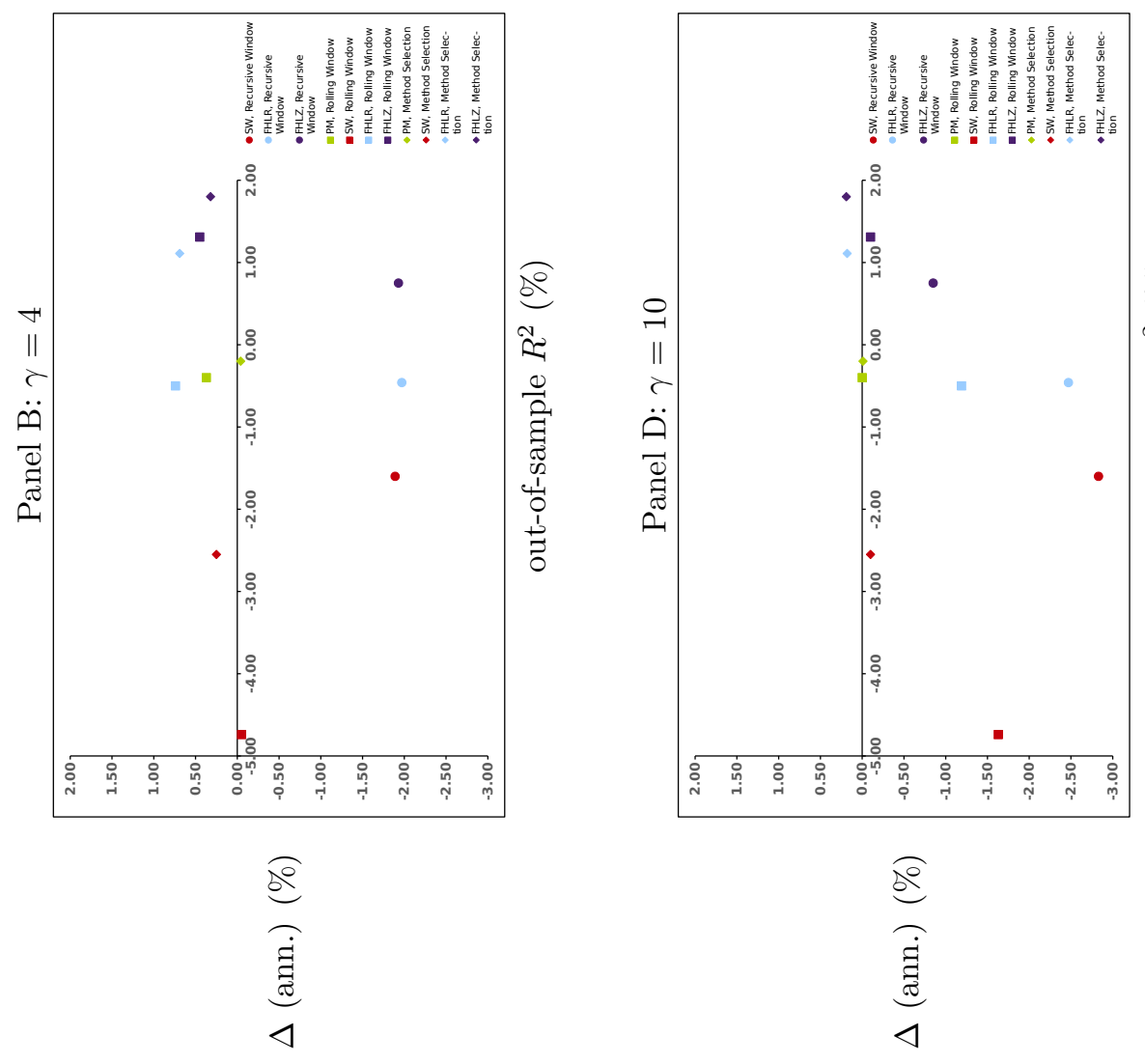

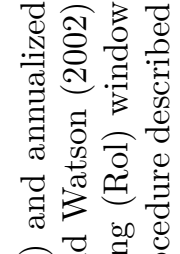

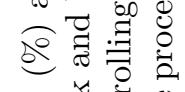

군

o 0 范

ڤँ

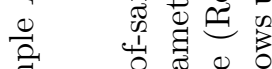

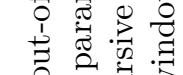

$\circ \approx \exists$ 3. 记 ชิ

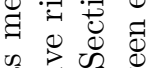
of.

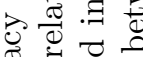

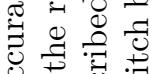

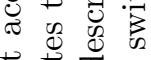
范

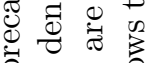
두워 范市总

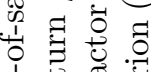
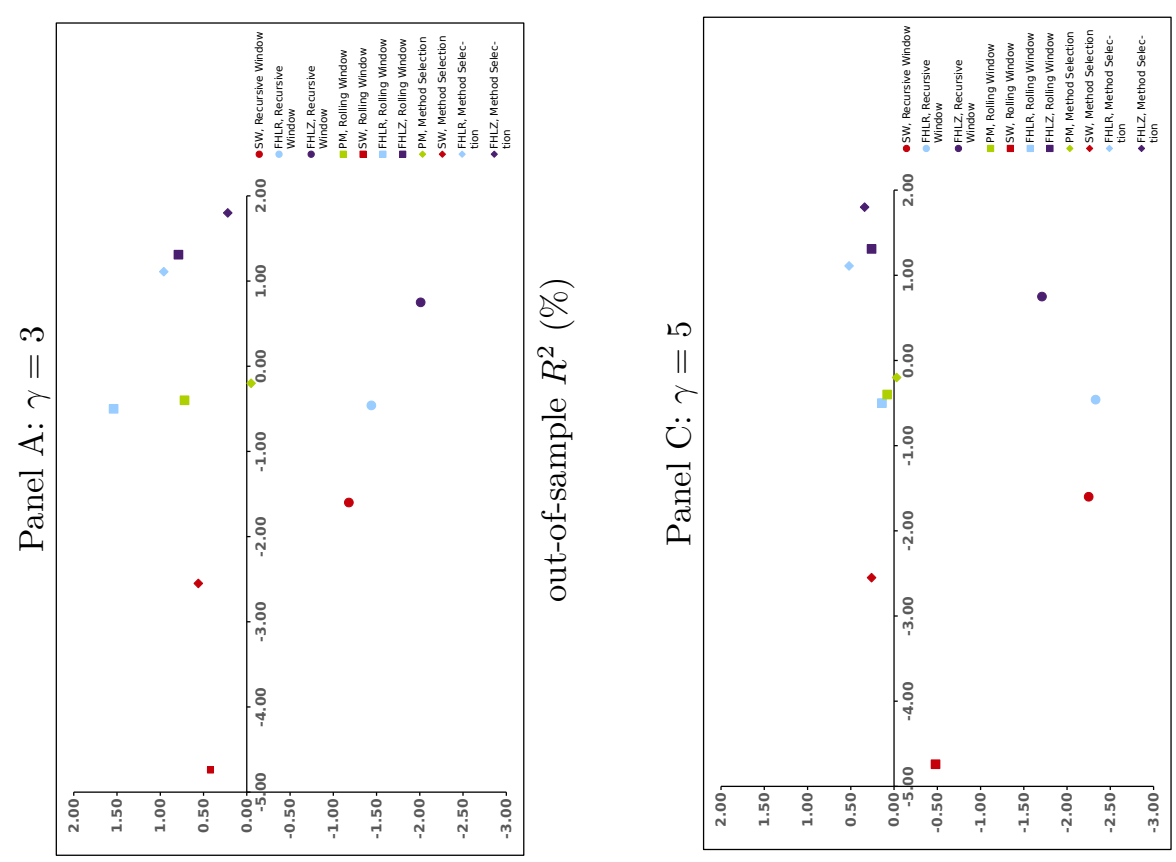

б 讨 겁

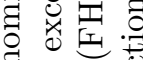
궁

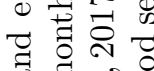
ब 95

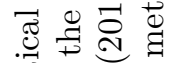

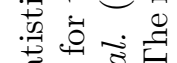

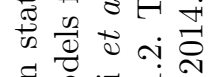

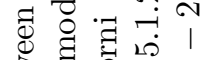
홍도용요

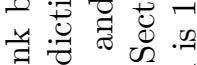
手乎 $=$

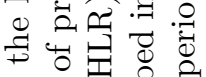

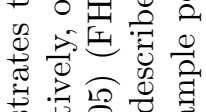

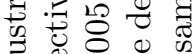

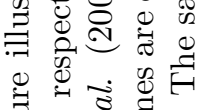

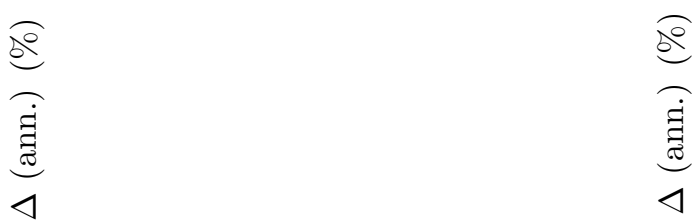

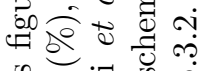

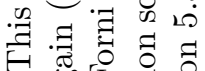
憨

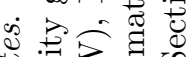

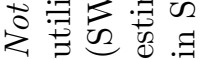


Figure 3: GR test: SW vs PM benchmark

FRED-MD
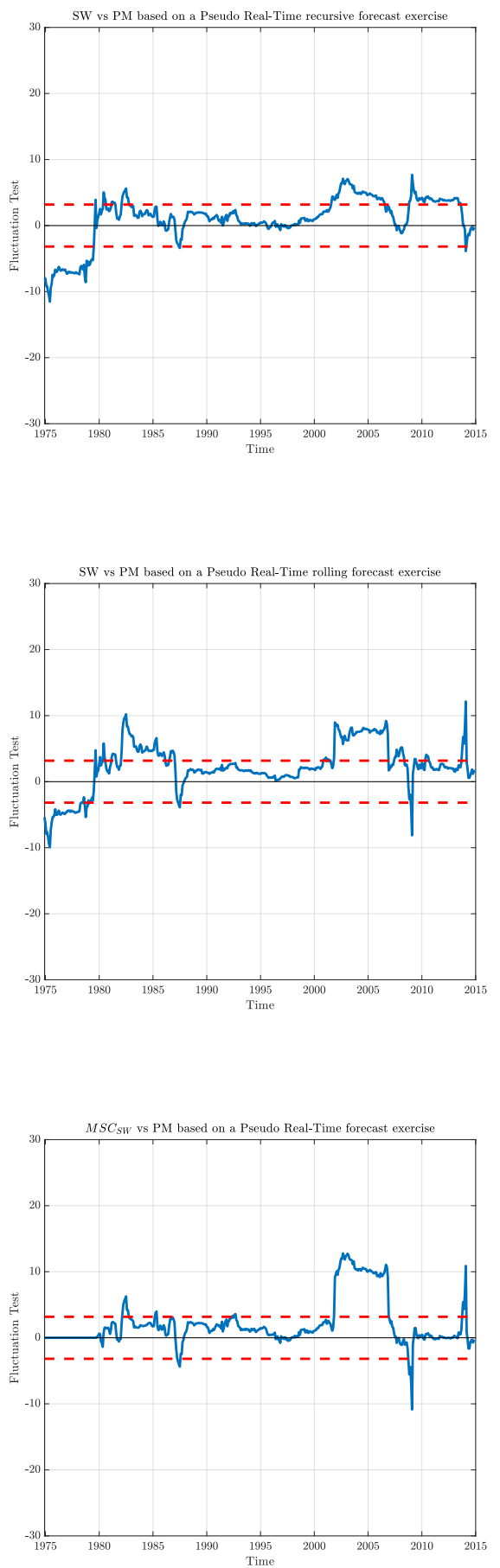

ALFRED
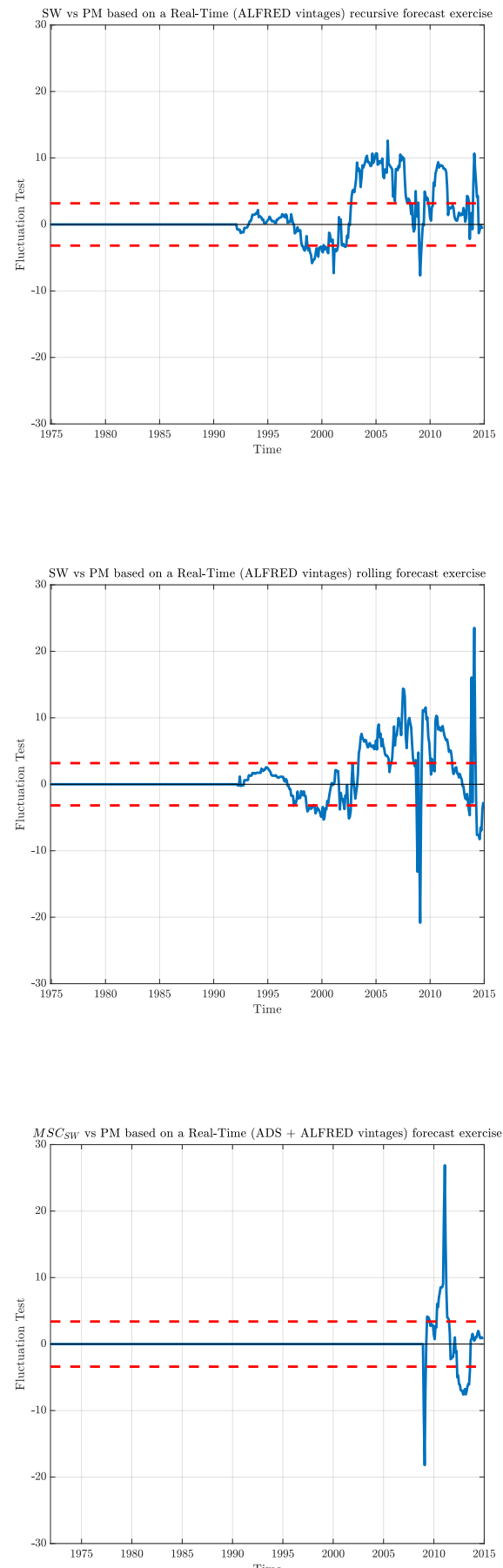

Notes. Forecasting methods are: recursive (top), rolling (centre) and method selection (bottom). GR test statistics (blue) within $5 \%$ confidence bands (red dashed); the smoothing adopted is 60 data points for FRED-MD data and 24 data points for ALFRED data. 
Figure 4: GR test: FHLR vs PM benchmark.

FRED-MD
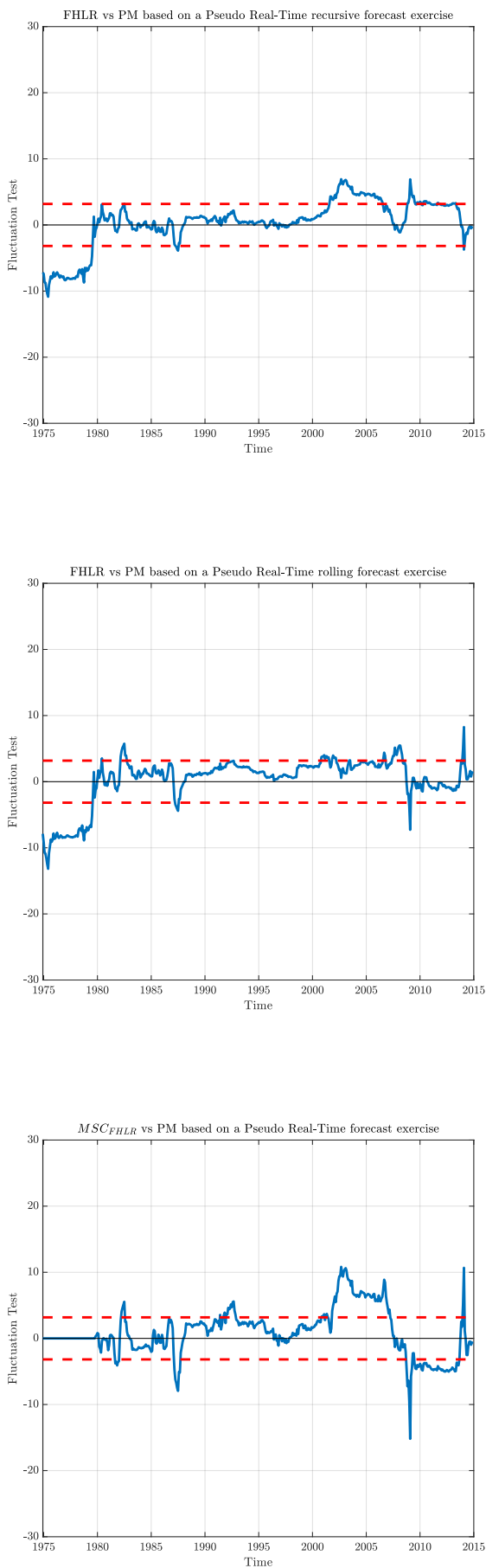

ALFRED
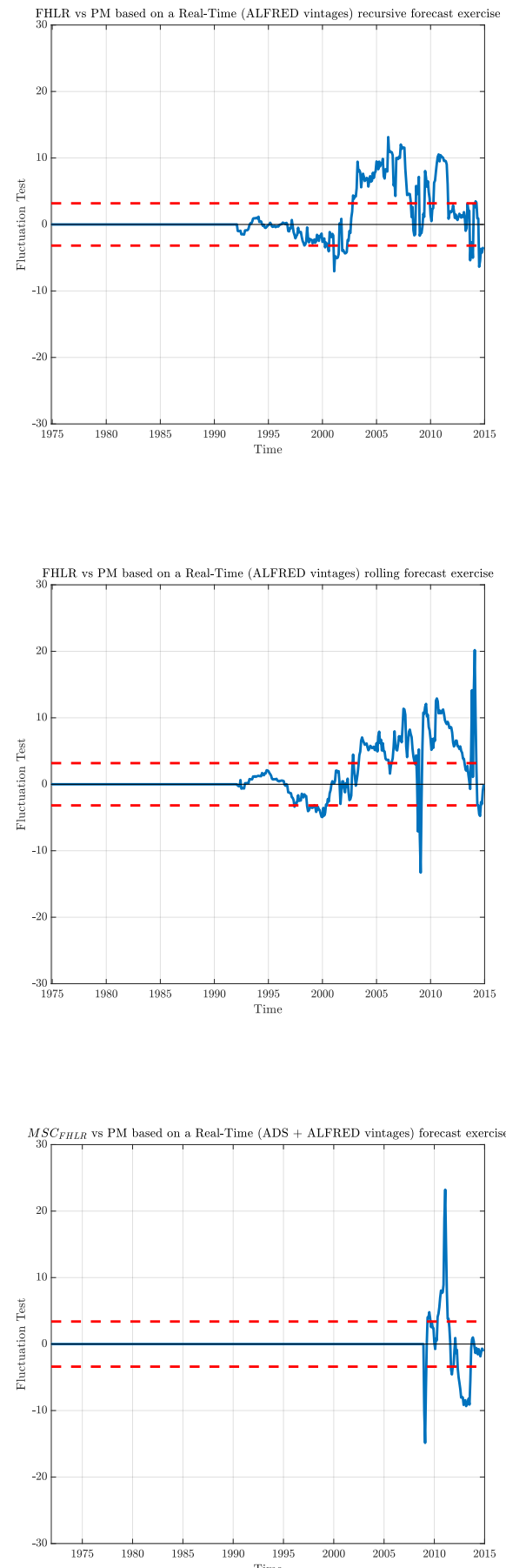

Notes. Forecasting methods are: recursive (top), rolling (centre) and method selection (bottom). GR test statistics (blue) within $5 \%$ confidence bands (red dashed); the smoothing adopted is 60 data points for FRED-MD data and 24 data points for ALFRED data. 
Figure 5: GR test: FHLZ vs PM benchmark

FRED-MD
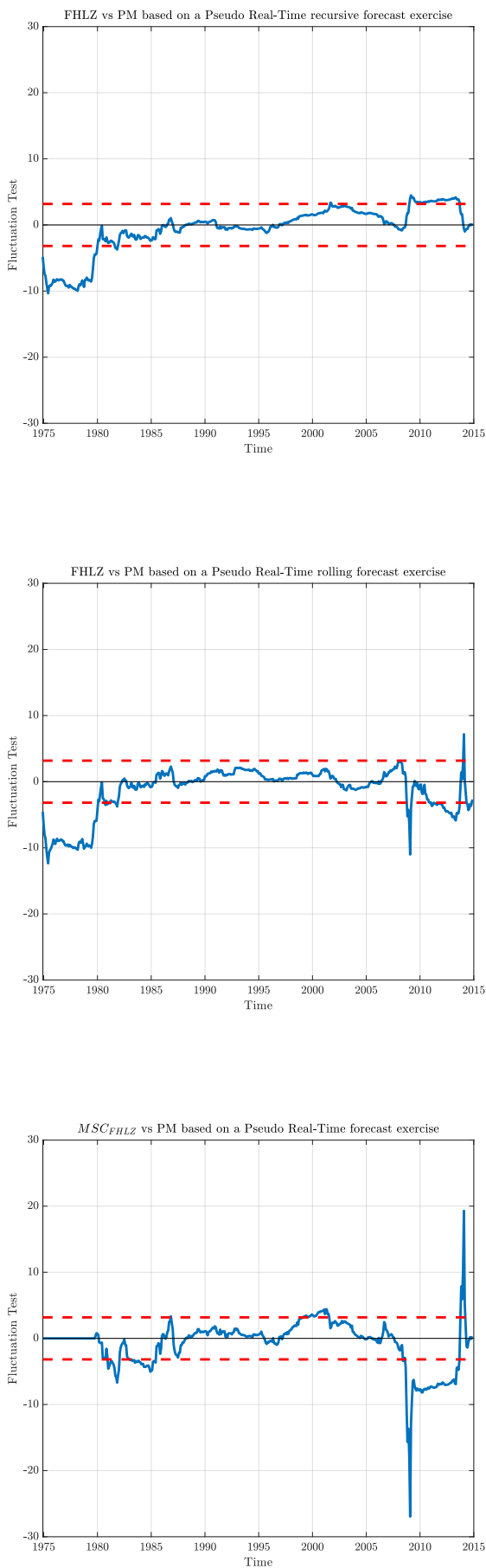

ALFRED
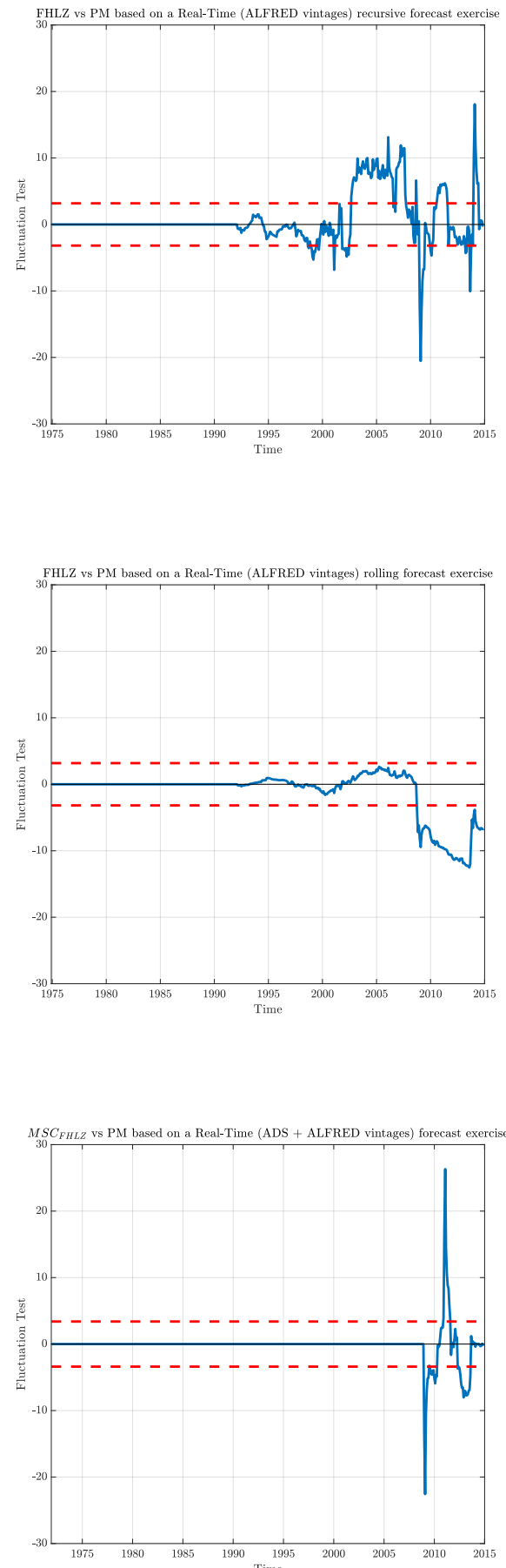

Notes. Forecasting methods are: recursive (top), rolling (centre) and method selection (bottom). GR test statistics (blue) within $5 \%$ confidence bands (red dashed); the smoothing adopted is 60 data points for FRED-MD data and 24 data points for ALFRED data. 


\section{Appendix: FRED-MD and ALFRED data}

We adopt the balanced version of FRED-MD dataset discarding the series with missing values at beginning of the sample. These are: PERMIT, PERMITNE, PERMITMW, PERMITS, PERMITW, ACOGNO, ANDENOx, TWEXMMTH, UMCSENTX.

Letting $x_{t}$ be a raw series, the transformations adopted are:

(1) no transformation;

(2) $\Delta x_{t}$;

(3) $\Delta^{2} x_{t}$;

(4) $\Delta \log \left(x_{t}\right)$;

(5) $\log \left(x_{t}\right)$;

(6) $\Delta^{2} \log \left(x_{t}\right)$

(7) $\Delta\left(\frac{x_{t}}{x_{t-1}-1}\right) \log \left(x_{t}\right)$;

Table A1: List of FRED-MD time series

\begin{tabular}{|c|c|c|c|}
\hline & mnemonic & description & tcode \\
\hline 1 & RPI & Real Personal Income & 5 \\
\hline 2 & W875RX1 & RPI ex. Transfers & 5 \\
\hline 3 & INDPRO & IP Index & 5 \\
\hline 4 & IPFPNSS & IP: Final Products and Supplies & 5 \\
\hline 5 & IPFINAL & IP: Final Products & 5 \\
\hline 6 & IPCONGD & IP: Consumer Goods & 5 \\
\hline 7 & IPDCONGD & IP: Durable Consumer Goods & 5 \\
\hline 8 & IPNCONGD & IP: Nondurable Consumer Goods & 5 \\
\hline 9 & IPBUSEQ & IP: Business Equipment & 5 \\
\hline 10 & IPMAT & IP: Materials & 5 \\
\hline 11 & IPDMAT & IP: Durable Materials & 5 \\
\hline 12 & IPNMAT & IP: Nondurable Materials & 5 \\
\hline 13 & IPMANSICS & IP: Manufacturing & 5 \\
\hline 14 & IPB51222S & IP: Residential Utilities & 5 \\
\hline 15 & IPFUELS & IP: Fuels & 5 \\
\hline 16 & NAPMPI & ISM Manufacturing: Production & 1 \\
\hline 17 & CAPUTLB00004S & Capacity Utilization: Manufacturing & 2 \\
\hline 1 & HWI & Help-Wanted Index for US & 2 \\
\hline 2 & HWIURATIO & Help Wanted to Unemployed ratio & 2 \\
\hline 3 & CLF16OV & Civilian Labor Force & 5 \\
\hline 4 & CE16OV & Civilian Employment & 5 \\
\hline 5 & UNRATE & Civilian Unemployment Rate & 2 \\
\hline 6 & UEMPMEAN & Average Duration of Unemployment & 2 \\
\hline 7 & UEMPLT5 & Civilians Unemployed $<5$ Weeks & 5 \\
\hline 8 & UEMP5TO14 & Civilians Unemployed 5-14 Weeks & 5 \\
\hline 9 & UEMP15OV & Civilians Unemployed >15 Weeks & 5 \\
\hline 10 & UEMP15T26 & Civilians Unemployed 15-26 Weeks & 5 \\
\hline 11 & UEMP $27 O V$ & Civilians Unemployed $>27$ Weeks & 5 \\
\hline 12 & CLAIMSx & Initial Claims & 5 \\
\hline 13 & PAYEMS & All Employees: Total nonfarm & 5 \\
\hline 14 & USGOOD & All Employees: Goods-Producing & 5 \\
\hline 15 & CES1021000001 & All Employees: Mining and Logging & 5 \\
\hline 16 & USCONS & All Employees: Construction & 5 \\
\hline 17 & MANEMP & All Employees: Manufacturing & 5 \\
\hline 18 & DMANEMP & All Employees: Durable goods & 5 \\
\hline 19 & NDMANEMP & All Employees: Nondurable goods & 5 \\
\hline 20 & SRVPRD & All Employees: Service Industries & 5 \\
\hline 21 & USTPU & All Employees: TT\&U & 5 \\
\hline 22 & USWTRADE & All Employees: Wholesale Trade & 5 \\
\hline 23 & USTRADE & All Employees: Retail Trade & 5 \\
\hline 24 & USFIRE & All Employees: Financial Activities & 5 \\
\hline 25 & USGOVT & All Employees: Government & 5 \\
\hline 26 & CES0600000007 & Hours: Goods-Producing & 1 \\
\hline 27 & AWOTMAN & Overtime Hours: Manufacturing & 2 \\
\hline 28 & AWHMAN & Hours: Manufacturing & 1 \\
\hline 29 & NAPMEI & ISM Manufacturing: Employment & 1 \\
\hline 30 & CES0600000008 & Ave. Hourly Earnings: Goods & 6 \\
\hline 31 & CES2000000008 & Ave. Hourly Earnings: Construction & 6 \\
\hline 32 & CES3000000008 & Ave. Hourly Earnings: Manufacturing & 6 \\
\hline 1 & HOUST & Starts: Total & 4 \\
\hline 2 & HOUSTNE & Starts: Northeast & 4 \\
\hline 3 & HOUSTMW & Starts: Midwest & 4 \\
\hline 4 & HOUSTS & Starts: South & 4 \\
\hline
\end{tabular}


Table A1 - continued from previous page

\begin{tabular}{|c|c|c|c|}
\hline 5 & HOUSTW & Starts: West & 4 \\
\hline 1 & DPCERA3M086SBEA & Real PCE & 5 \\
\hline 2 & CMRMTSPLx & Real M\&T Sales & 5 \\
\hline 3 & RETAILx & Retail and Food Services Sales & 5 \\
\hline 4 & NAPM & ISM: PMI Composite Index & 1 \\
\hline 5 & NAPMNOI & ISM: New Orders Index & 1 \\
\hline 6 & NAPMSDI & ISM: Supplier Deliveries Index & 1 \\
\hline 7 & NAPMII & ISM: Inventories Index & 1 \\
\hline 8 & AMDMNOx & Orders: Durable Goods & 5 \\
\hline 9 & AMDMUOx & UnPlled Orders: Durable Goods & 5 \\
\hline 10 & BUSINVx & Total Business Inventories & 5 \\
\hline 11 & ISRATIOx & Inventories to Sales Ratio & 2 \\
\hline 1 & M1SL & M1 Money Stock & 6 \\
\hline 2 & M2SL & M2 Money Stock & 6 \\
\hline 3 & M3SL & MABMM301USM189S in FRED, M3 for the United States & 6 \\
\hline 4 & M2REAL & Real M2 Money Stock & 5 \\
\hline 5 & AMBSL & St. Louis Adjusted Monetary Base & 6 \\
\hline 6 & TOTRESNS & Total Reserves & 6 \\
\hline 7 & NONBORRES & Nonborrowed Reserves & 6 \\
\hline 8 & BUSLOANS & Commercial and Industrial Loans & 6 \\
\hline 9 & REALLN & Real Estate Loans & 1 \\
\hline 10 & NONREVSL & Total Nonrevolving Credit & 6 \\
\hline 11 & CONSPI & Credit to PI ratio & 2 \\
\hline 12 & MZMSL & MZM Money Stock & 6 \\
\hline 13 & DTCOLNVHFNM & Consumer Motor Vehicle Loans & 6 \\
\hline 14 & DTCTHFNM & Total Consumer Loans and Leases & 6 \\
\hline 15 & INVEST & Securities in Bank Credit & 6 \\
\hline 1 & FEDFUNDS & Effective Federal Funds Rate & 2 \\
\hline 2 & CP3M & 3-Month AA Comm. Paper Rate & 2 \\
\hline 3 & TB3MS & 3-Month T-bill & 2 \\
\hline 4 & TB6MS & 6-Month T-bill & 2 \\
\hline 5 & GS1 & 1-Year T-bond & 2 \\
\hline 6 & GS5 & 5-Year T-bond & 2 \\
\hline 7 & GS10 & 10-Year T-bond & 2 \\
\hline 8 & AAA & Aaa Corporate Bond Yield & 2 \\
\hline 9 & BAA & Baa Corporate Bond Yield & 2 \\
\hline 10 & COMPAPFF & CP - FFR spread & 1 \\
\hline 11 & TB3SMFFM & 3 Mo. - FFR spread & 1 \\
\hline 12 & TB6SMFFM & 6 Mo. - FFR spread & 1 \\
\hline 13 & T1YFFM & 1 yr. - FFR spread & 1 \\
\hline 14 & T5YFFM & 5 yr. - FFR spread & 1 \\
\hline 15 & T10YFFM & 10 yr. - FFR spread & 1 \\
\hline 16 & AAAFFM & Aaa - FFR spread & 1 \\
\hline 17 & BAAFFM & Baa - FFR spread & 1 \\
\hline 18 & EXSZUS & Switzerland / U.S. FX Rate & 5 \\
\hline 19 & EXJPUS & Japan / U.S. FX Rate & 5 \\
\hline 20 & EXUSUK & U.S. / U.K. FX Rate & 5 \\
\hline 21 & EXCAUS & Canada / U.S. FX Rate & 5 \\
\hline 1 & PPIFGS & PPI: Finished Goods & 6 \\
\hline 2 & PPIFCG & PPI: Finished Consumer Goods & 6 \\
\hline 3 & PPIITM & PPI: Intermediate Materials & 6 \\
\hline 4 & PPICRM & PPI: Crude Materials & 6 \\
\hline 5 & oilprice & Crude Oil Prices: WTI & 6 \\
\hline 6 & PPICMM & PPI: Commodities & 6 \\
\hline 7 & NAPMPRI & ISM Manufacturing: Prices & 1 \\
\hline 8 & CPIAUCSL & CPI: All Items & 6 \\
\hline 9 & CPIAPPSL & CPI: Apparel & 6 \\
\hline 10 & CPITRNSL & CPI: Transportation & 6 \\
\hline 11 & CPIMEDSL & CPI: Medical Care & 6 \\
\hline 12 & CUSR0000SAC & CPI: Commodities & 6 \\
\hline 13 & CUUR0000SAD & CPI: Durables & 6 \\
\hline 14 & CUSR0000SAS & CPI: Services & 6 \\
\hline 15 & CPIULFSL & CPI: All Items Less Food & 6 \\
\hline 16 & CUUR0000SA0L2 & CPI: All items less shelter & 6 \\
\hline 17 & CUSR0000SA0L5 & CPI: All items less medical care & 6 \\
\hline 18 & PCEPI & PCE: Chain-type Price Index & 6 \\
\hline 19 & DDURRG3M086SBEA & PCE: Durable goods & 6 \\
\hline 20 & DNDGRG3M086SBEA & PCE: Nondurable goods & 6 \\
\hline 21 & DSERRG3M086SBEA & PCE: Services & 6 \\
\hline 1 & S\&P 500 & S\&P: Composite & 5 \\
\hline 2 & S\&P: indust & S\&P: Industrials & 5 \\
\hline 3 & $\mathrm{~S} \& \mathrm{P}$ div yield & S\&P: Dividend Yield & 2 \\
\hline 4 & $\mathrm{~S} \& \mathrm{P}$ PE ratio & S\&P: Price-Earnings Ratio & 5 \\
\hline
\end{tabular}


Table A2: List of ALFRED time series

\begin{tabular}{|c|c|c|c|}
\hline Mnemonic & Variable Description & TCode & Start Date \\
\hline AWHMAN & Avg Weekly Hours of Production and Nonsupervisory Employees: Manufacturing & 4 & $11 / 1 / 1964$ \\
\hline AWHNONAG & Avg Weekly Hours Of Production And Nonsupervisory Employees: Total private & 4 & $5 / 1 / 1970$ \\
\hline AWOTMAN & Avg Weekly Overtime Hours of Production and Nonsupervisory Employees: Manufacturing & 4 & $8 / 1 / 1966$ \\
\hline CE16OV & Civilian Employment & 4 & $12 / 1 / 1964$ \\
\hline CLF16OV & Civilian Labor Force & 4 & $11 / 1 / 1964$ \\
\hline CPIAUCSL & Consumer Price Index for All Urban Consumers: All Items & 4 & $6 / 1 / 1972$ \\
\hline CURRDD & Currency Component of M1 Plus Demand Deposits & 4 & $11 / 1 / 1964$ \\
\hline CURRSL & Currency Component of M1 & 4 & $11 / 1 / 1964$ \\
\hline DEMDEPSL & Demand Deposits at Commercial Banks & 4 & $9 / 1 / 1964$ \\
\hline DMANEMP & All Employees: Durable goods & 4 & $11 / 1 / 1964$ \\
\hline DSPI & Disposable Personal Income & 4 & $1 / 1 / 1980$ \\
\hline DSPIC96 & Real Disposable Personal Income & 4 & $2 / 1 / 1980$ \\
\hline HOUST & Housing Starts: Total: New Privately Owned Housing Units Started & 4 & $12 / 1 / 1964$ \\
\hline HOUST1F & Privately Owned Housing Starts: 1-Unit Structures & 4 & $2 / 1 / 1972$ \\
\hline HOUST2F & Housing Starts: 2-4 Units & 4 & $2 / 1 / 1973$ \\
\hline INDPRO & Industrial Production Index & 4 & $11 / 1 / 1964$ \\
\hline M1SL & M1 Money Stock & 4 & $12 / 1 / 1979$ \\
\hline M2SL & M2 Money Stock & 4 & $12 / 1 / 1979$ \\
\hline MANEMP & All Employees: Manufacturing & 4 & $11 / 1 / 1964$ \\
\hline NDMANEMP & All Employees: Nondurable goods & 4 & $11 / 1 / 1964$ \\
\hline OCDSL & Other Checkable Deposits & 4 & $2 / 1 / 1981$ \\
\hline PAYEMS & All Employees: Total nonfarm & 4 & $11 / 1 / 1964$ \\
\hline $\mathrm{PCE}$ & Personal Consumption Expenditures & 4 & $12 / 1 / 1979$ \\
\hline PCEC96 & Real Personal Consumption Expenditures & 4 & $3 / 1 / 1980$ \\
\hline PCEDG & Personal Consumption Expenditures: Durable Goods & 4 & $12 / 1 / 1979$ \\
\hline PCEDGC96 & Real Personal Consumption Expenditures: Durable Goods & 4 & $3 / 1 / 1980$ \\
\hline PCEND & Personal Consumption Expenditures: Nondurable Goods & 4 & $12 / 1 / 1979$ \\
\hline PCENDC96 & Real Personal Consumption Expenditures: Nondurable Goods & 4 & $3 / 1 / 1980$ \\
\hline PCES & Personal Consumption Expenditures: Services & 4 & $12 / 1 / 1979$ \\
\hline PCESC96 & Real Personal Consumption Expenditures: Services & 4 & $3 / 1 / 1980$ \\
\hline PFCGEF & Producer Price Index: Finished Consumer Goods Excluding Foods & 4 & $1 / 1 / 1982$ \\
\hline PI & Personal Income & 4 & $2 / 1 / 1966$ \\
\hline PPICFF & Producer Price Index: Crude Foodstus $\backslash \&$ Feedstus & 4 & $1 / 1 / 1982$ \\
\hline PPICPE & Producer Price Index: Finished Goods: Capital Equipment & 4 & $1 / 1 / 1978$ \\
\hline PPICRM & Producer Price Index: Crude Materials for Further Processing & 4 & $3 / 1 / 1978$ \\
\hline PPIFCF & Producer Price Index: Finished Consumer Foods & 4 & $1 / 1 / 1982$ \\
\hline PPIFGS & Producer Price Index: Finished Goods & 4 & $1 / 1 / 1982$ \\
\hline PPIIFF & Producer Price Index: Intermediate Foods $\backslash \&$ Feeds & 4 & $1 / 1 / 1982$ \\
\hline PPIITM & Producer Price Index: Intermediate Materials: Supplies $\backslash \&$ Components & 4 & $3 / 1 / 1978$ \\
\hline SAVINGSL & Savings Deposits - Total & 4 & $12 / 1 / 1979$ \\
\hline SRVPRD & All Employees: Service-Providing Industries & 4 & $9 / 1 / 1971$ \\
\hline STDCBSL & Small Time Deposits at Commercial Banks & 4 & $12 / 1 / 1979$ \\
\hline STDSL & Small Time Deposits - Total & 4 & $12 / 1 / 1979$ \\
\hline STDTI & Small Time Deposits at Thrift Institutions & 4 & $12 / 1 / 1979$ \\
\hline SVGCBSL & Savings Deposits at Commercial Banks & 4 & $12 / 1 / 1979$ \\
\hline SVGTI & Savings Deposits at Thrift Institutions & 4 & $12 / 1 / 1979$ \\
\hline SVSTCBSL & Savings and Small Time Deposits at Commercial Banks & 4 & $12 / 1 / 1979$ \\
\hline SVSTSL & Savings and Small Time Deposits - Total & 4 & $12 / 1 / 1979$ \\
\hline TCDSL & Total Checkable Deposits & 4 & $3 / 1 / 1981$ \\
\hline UEMP15OV & Civilians Unemployed - 15 Weeks $\backslash \&$ Over & 4 & $11 / 1 / 1964$ \\
\hline UEMP15T26 & Civilians Unemployed for 15-26 Weeks & 4 & $1 / 1 / 1982$ \\
\hline UEMP27OV & Civilians Unemployed for 27 Weeks and Over & 4 & $1 / 1 / 1966$ \\
\hline UEMP5TO14 & Civilians Unemployed for 5-14 Weeks & 4 & $11 / 1 / 1964$ \\
\hline UEMPLT5 & Civilians Unemployed - Less Than 5 Weeks & 4 & $11 / 1 / 1964$ \\
\hline UEMPMEAN & Average (Mean) Duration of Unemployment & 4 & $1 / 1 / 1972$ \\
\hline UEMPMED & Median Duration of Unemployment & 4 & $1 / 1 / 1982$ \\
\hline UNEMPLOY & Unemployed & 4 & $12 / 1 / 1964$ \\
\hline UNRATE & Civilian Unemployment Rate & 2 & $2 / 1 / 1960$ \\
\hline USCONS & All Employees: Construction & 4 & $12 / 1 / 1964$ \\
\hline USFIRE & All Employees: Financial Activities & 4 & $12 / 1 / 1964$ \\
\hline USGOOD & All Employees: Goods-Producing Industries & 4 & 9/1/1971 \\
\hline USGOVT & All Employees: Government & 4 & $12 / 1 / 1964$ \\
\hline USMINE & All Employees: Mining and logging & 4 & $12 / 1 / 1964$ \\
\hline USPRIV & All Employees: Total Private Industries & 4 & $8 / 1 / 1971$ \\
\hline USSERV & All Employees: Other Services & 4 & $12 / 1 / 1964$ \\
\hline USTPU & All Employees: Trade, Transportation $\backslash \&$ Utilities & 4 & $12 / 1 / 1964$ \\
\hline USTRADE & All Employees: Retail Trade & 4 & $12 / 1 / 1964$ \\
\hline USWTRADE & All Employees: Wholesale Trade & 4 & $12 / 1 / 1964$ \\
\hline
\end{tabular}

